\title{
Kulturelle Grenzen in der Expansion Europas
}

\author{
Von \\ JURGEN OSTERHAMMEI. \\ Hagen
}

1. Kulturkontakt'

Die frühen europäischen „Entdecker" der überseeischen Welt und die philosophischeren unter ihren Eroberern, Hernán Cortés zum Beispiel, waren sich der Tatsache bewußt, nicht auf transhumane Fabelwesen oder auf unstrukturierte Primitivität gestoßen zu sein, sondern auf menschliche Kollektive, deren I bensführung Zusammenhang und Regelhaftigkeit erkennen ließ, kurz: auf andere Kulruren. ${ }^{2}$ Manche von ihnen erkannten auch die strukturelle Verwandischaft zwischen ihrer jeweiligen Heimargesellschaft und nicht wenigen Gemeinwesen, auf die sie in Ubersee stießen. So war die vertikale Gliederung und Statusdifferenzierung der Aztekengesellschaft von der im Spanien Karls V. durchaus nicht fundamental verschieden. Gewiß, erst Gelehrte der Hochrenaissance wie José de Acosta, der Verfasser einer enzyklopädischen „Historia natural y moral de las Indias" (vollender 1590) und Matteo Ricci, der erste unbefangen analysierende europäische Beobachter der chinesischen Zivilisation, ${ }^{3}$ vermochten den systemischen Zusammenhang in eimer nichteuropaischen Kultur zu erkennen und

1 Eine frühere Fassung dieses Aufsatzes wurde 1992 auf dem Historikeriag in Hannover vorgerragen. Zahlreiche Anregungen verdanke ich den Symposien au univers.lhistorischen Fragen. die das BreuningerKolleg Sturtgart 1990 bis 1994 jährlich aen 1itisee veranstaleere, insbesondere der Tagung des Jahres 1992. Kolleg Sturtgatt
die sich mir dem Thema „Die Begegnung mir dern Fremder" beschäfigie. An den litiser-Kolloquien nahm

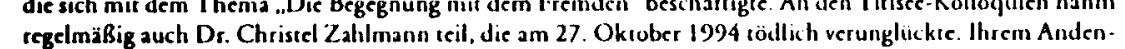
regelmäß̈ig auch Ds. Christel Zathlma
ken soll der Aufsarz gewidmet sein.

ken soll der Aufsarz gewidmet sein.
2 lm folgenden wird, ohne cinen cigenen Definitionsversuch zu unternehmen, ein weirgefaßter nicht-normativer und pluraler Begriff von Kulcur/Kulturen im Sinne der Anthropologie verwender, genauer: im Sinne der dritten von drei Hauperichtungen des Verständnisses von .Kultur”: a) als eine Summe von Wissen, b) als Ensemble von Inscitutionen, c) als kollektiv entworfener, verbindlicher Sinnkosmos. Diese Unterscheidung nach Roy G. D'Andrade. Cultural Meaning Systems, in: Richard A. Shweder/Robert A. LeVine (Hrsg.). Culture Theory: Essays on Mind, Self, and Emotion (Cambridge 1984) 88-119, hier 115. ,Zivilisation" wird davon nicht terminologisch scharf unterschieden. Vgl. dazu als begriffsgeschichtliche Begründung Jörg Fisch, „Zivilisarion, Kultur", in: Oro Brunner/Werner Conze/Reinhast Koselleck (Hrsg.). Ge-
schicheliche Grundbegriffe. Historisches Lexikon zur politisch-sozialen Sprache in Deutschland. Bd. 7 schicheliche Grundbegriffe. Historisches
(Stuttgart 1992) 679-774, bes. 771 f.

(Sturtgart 1992) 679-774, bes. 771f. 1962). Vgl. zum Werk und seinem Autor: Authony Pagden, The liall of Natural Man: The American Indian and the Origins of Comparative Ethnology (Cambridge 1982) 146-197. Die maßgetende Textgrundlage fur Ricci ist Pasquale d'Llia (Hrsg). Fonci Ricciane. Documenti originali concernanci Marteo Ricci c la storia delle prime Relazioni era l'Europa e la Cina (1579-1615). 3 Bde. (Rom 1942-1949). Vgl. insbes. David E. Mungello, Curious Land: Jesuit Accomodation and the Origins of Sinology (Wiesbadicn 1985) 44-73: Jonachan D. Spence, The Metnory Paiace of Matteu Ricci (London 1985). 
wurden so zu Vorläufern oder Begründern einer komparativen Ethnologie. ${ }^{4}$ Aber schon aus den ersten Kontakterfahrungen entstand vielfach der Eindruck, daß die Fremdartigkeit der Anderen nicht allein eine Zerrspiegelung des Eigenen sei, nicht bloß Ausdruck einer absurd verkehrten, die natürliche Ordnung pervertierenden Welt, daß sie vielmehr mit durchaus komplexen Sinngebungen verbunden war. Wie diese zu bewerten seien, blieb eine zweite Frage, die viel kontroverser war und die von Anfang an in der Spannung zwischen Relativismus und Universalismus strittig beantwortet wurde. So ließen sich, um ein besonders spektakuläres Beispiel zu nennen, die Menschenopfer der Azteken schon im 16. Jahrhundert nicht nur als Verbrechen, die „über das Maß an jeder menschlichen Verderbtheit hinausgehen" und hinreichende Gründe für einen Unterwerfungskrieg lieferten, interprerieren (Juan Ginés de Sepúlveda), ${ }^{5}$ sondern auch nachsichtiger als ein eher auf Verstandesträgheit zurückführbares Mißverständnis des göttlichen Naturgesetzes (Francisco de Vitoria) ${ }^{6}$ oder gar geradezu verständnisvoll als "wenig mehr denn irregeleitete Frömmigkeit" (Bartolomé de las Casas). ${ }^{\text {? }}$

Um die fremden Sinnwelten zu erschließen, wandıen die Europäer in vielen Varianten eine Art von transkultureller Hermeneutik an. Diese war selten interesselos-akademisch. Sie konnte in verschiedenartigen Situationen unterschiedlichen Zwecken dienen. Der offensichılichste war die Eroberung. Hernán Cortés, wie ihn Tzvetan Todorov in einer einflußreichen, aber vermulich die Naivität seiner Gegenspieler übertreibenden Studie porträtiert hat, vermochte die militärische Schwäche seine Expeditionstruppe gegenüber der Azrekenmacht dadurch zu kompensieren, daß er urteilstrübende und handlungshenmende Rigiditaten im Weltubild der Mexica durch ein virtuoses Spiel mit Symbolen für sein unbeirtt verfolgtes Eroberungsziel instrumentalisierte. ${ }^{8}$ Missionare gingen bei der spirituellen Seelennahme behutsamer, aber im Prinzip ähnlich vor. Ihr vornehmstes Medium war die Sprache. Sie erlernten einheimische Idiome, um sich in ihnen predigend verständlich machen und, unterstützt durch den Einsatz bildlichen Materials, ein religiöses Deutungsmonopol erlangen zu können. ${ }^{9}$ Dort, wo sie, wie in Japan und insbesondere in China, auf widerständige Elitekulturen stießen, unternahmen sie heroische Anstrengungen philologischer Ge-

\footnotetext{
4 Es soll hier sellhstverständlich nicht behaupter werden, eine solche vormoderne Ethnographie und Eth nologie sei cine besondere Errungenschaft Europas. Es gab sie zurnindest auch in China und vor allem in der arabisch-istamischen Literatur des Mittclaleers. Zu dieser vgl. Aziz Al-Azmeh, Barbarians in Arab Eyes, in: Past \& Present 134 (Februar 1992) 3-18.

5 Juan Ginés de Sepúlveda, Democrates segundo o De las justas causas de la guerra contra los Indios Iverfaßt 15461 zir nach Chrissoph Scroserzki (Hirsg). Der Griff nach der Neuen Wels. Der Untergang der ca. TS4A). zic Ku he Che

Vgl. Pagden (wic Anm. 3) 90

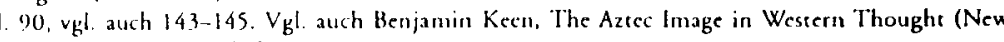
Buswiek. N. J. (1971) Kap. 4-6

8 Vgl. Teveran Tidorov, Die Lioberung Amerikas. Das Drobtem des Anderen (1:rankfurt a. M. 1985, zuerst frz. 1982) Gyff

Brillant dazu: Serge Gruzinski, La colonisation de l'imaginairc: Sociérés indigènes et occidentalisation
} dans le Mcxique espagnol XVle-XVIlle sičcle (Paris 1988) is

lehrsamkeit mit dem Ziel, durch allmähliche „Akkomodation“ Elemente des christlichen Glaubens in die Diskurse der selbstbewußten Gastzivilisationen einzuschmuggeln. ${ }^{10}$ Meist, aber keineswegs in jedem Falle gelang es, die hermeneutischen Vorteile des Europäers, der mit sparsamstem Mitteleinsatz stets Herr der Situation bleiben sollte, erfolgreich auszuspielen. Der Tod von Kapitan James Cook an der KealakekuaBucht am 14. Februar 1779 ist, selbst wenn man einigen der Einwände gegen Marshall Sahlins' berühmte Interpretation folgt, ohne Zweifel durch Fehlinterpretationen hawaiianischer „Zeichen“ ausgelöst worden, die dem sonst so urteilssicheren EntdeckerEthnographen unterliefen. "Cook wurde das Opfer einer kulturwissenschaftlichen Insuffizienz.

Es bedurfte also nicht erst des modernen anthropologischen Kulturbegriffs, der den distinkten Ensemblecharakter von Symbolen, Verhaltensregeln und materieller Existenzsicherung betont, um unter Europäern die Einsicht zu befördern, daß man nicht einem willkürlichen Chaos, sondern anderen Ordnungen menschlicher Lebensgestaltung konfrontiert war: seien es nun diejenigen von schriftlosen „Wilden“, schrifibesitzenden „Barbaren“ oder gar - wie im Falle der europäischen Japan- und phasenweise auch Chinaauffassung - andersartig "Zivilisierten“. Schon viele Prakriker der Expansion erkannten also, was ihre Historiker erst langsam lernen sollten: Die Ausbreitung von Europäern über den Globus führte zu Kulturkontakten mannigfaltiger Art. Die europäische Expansion, von ihren Formen und Wirkungen her betrachtet, war ein umfassendes Phänomen interkultureller Begegnung. ${ }^{12}$

Die Uberseegeschichtsschreibung hat diese beinahe trivial anmutende Einsicht lange Zeit nicht hinreichend gewürdigt. Als Entdeckungsgeschichte war sie auf die Taten heroischer Einzelner fixiert, als Kolonisationsgeschichte auf die Leistungen siedelnder und staatengründender Gemeinschaften, als Kolonialgeschichte auf politische Form und wirtschaftliche Funktionsweise von Kolonialreichen, als Imperialgeschichte auf die Auseinandersetzungen der Großen Mächte im Zuge der Globalisierung europäi-

10 Zur Bedcutung von Sprachstudien für die europäische Expansion vgl. Wolfgang Reinhard, Sprachbe herrschung und Weltherrschaft. Sprache und Sprachwissenschaft in der curopäischen Expansion, in: ders. (Hrsg.), Humanismus und neue Wele (Weinheim 1987) 1-36.

11 Marshall Sahlins, Der Tod des Kapitän Cook. Geschichee als Merapher und Mythos als Wirklichkeit in der Frühgeschichte des Königreichs Hawaii (Berlin 1986, zuerst amerik. 1981) 32ff.; zur Kricik daran vgl. vor allem Gananath Obeyesckere, The Apotheosis of Capeain Cook: European Mythmaking in the Pacific (Princeton, N. J. 1992) bes. 49-73. Allerdings hatten Cook und seine Begleiter die größten Schwierigkeiten der Kommunikation in der Begegnung mir Auseraliern und Tasmaniern, weniger mit den Bewohnern der Kon

12 Unter Expansion soll hier, dem üblichen Sprachgebrauch folyend, die übersecische Ausbreirung von Europiern seir Kolo in Peripherien maßgebende Bedeurung bei der homogenisierenden Fornienng des christlichen Luropa zukommt, ist neuerdings von Mediavisen bekrahge wo of Europe: Conquest, Colonization and Cultural Change, 950-1350 (London 1993). Vgl. auch die universalhistorische Interpretation mittclalterlicher Expansionsvorgänge bei Archibald R. Lewis, Nomads and Crusaders, A. D. 1000-1368 (Bloomington/hadianapolis 1988). 
scher Politik. Zuständig für Kulturelles war überwiegend die Missionsgeschichte, die jedoch bis in die 1970cr Jahre hinein einem engen Evangelisierungsparadigma verhaftet blieb und die über Christianisierung hinausweisenden Aspekte eines von Missionaren geplanten oder ungewollt in Gang gesetzten Kulturtransfers nur beiläufig behandelte. Von der neuen Differenziertheit, welche die Expansionsgeschichte vor etwa zwei Jahrzehnten zu erlangen begann, ${ }^{13}$ hat eine kulturgeschichtliche Perspektive bis vor kurzem, als die Analyse "kolonialer Diskurse“ in den Vordergrund trat, ${ }^{14}$ von allen Bereichen am wenigsten profitiert. Nur im Falle Lateinamerikas, wo seit den frühen achtziger Jahren der überkommenen Vorstellung von der "Entdeckung" mit politischem Nachdruck der Begriff der "Begegnung zweier Welten“ (encuentro de dos mundos) entgegengesetzt wurde, ${ }^{15}$ hat sich ein breiteres Arbeitsfeld interkultureller Beziehungsgeschichte entwickelt. Die Gründe für den relativen Rückstand liegen in der Widerspenstigkeit des Sache: Erstens fordert interkulturelle Beziehungsgeschichte im Idealfall von Historikerinnen und Historikern eine bikulturelle Kompetenz, das Vermögen also, die Perspektive nicht allein der Entdeckenden, sondern auch die der Entdeckten einzunehmen. ${ }^{16}$ Zweitens sind kulturelle Phänomene eigensinniger, lokaler, partikularer, schwieriger cinem sirukcurierenden Zugriff zu unierwerfen als etwa die Zusammenhange des incerkonunemeden Handels oder des organisatorischen Auf-

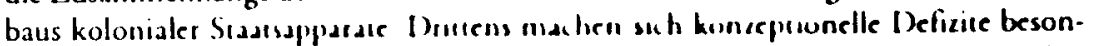

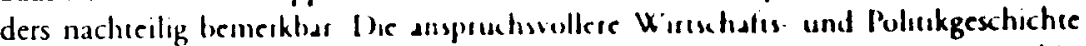
der europäischen Expansun kunn von enron ldrenilestand proficueren, der. gemeinhin unter dem Titel .Imperialismustheorien" subsumerl, sowolil .grobe“ Theorien der Weltentwicklung als auch - für Spezialfragen der Expansionsgeschichce - begrenzte-

13 Eine Summe dieser neueren Forschung ziche Wollgang Reinhard, Geschichte der europäischen Expan13 Eine Summe dieser neure sion. 4 Bde. (Sturtgart usw. 1983-190). Der Aur har au Missionsgeschichee als Kulturgeschichre gegeben: Gelenkter Kulturwandel im siebzehnten Jahthundert. Akkulturation in den jesuitenmissionen als universalhistorisches Problem, in: Historische Zeitschrift 223 (1976) 529-590.

14 Repräsentativ für unterschiedliche Ausprägungen dieser Interessenrichrung sind die Sammelbände Stephen Giecnblatt (Hirsg.). New World Eincounters (Berkeley/Los Angeles/Oxford 1993) und Carol A. Breckenridge/Peter van der Veer (Hrsg). Oriencalism and the Postcolonial Predicament: Perspectives on Souch Asiz (Philadelphia 1993). Der wichrigste theorerische Inspirator im Hintergrund ist im ersten Fall Michel de Certeau, im zweiten Edward Said.

is $\mathrm{Vgl}$. Horst Pietschmann, Die Iberische Expansion in Amerika in der neueren Forschungsdiskussion, in: Periplus, Jahrbuch für außercuropä ische Geschichte 2 (1992), 72-80, hier 72; Walther L. Bernecker, „Rau"eriplus. Jare" Das Jubizum der Enodeckung" Amerikas im Widerstreit der Meinungen, in: Wolfgang chender Spiegel. Das Juli der ..Endeck g̈̈ An Amerika Gemeinsamkeiten - Gegensäzze - europäiReinhard/leter Waldmann (Hisg.). Nord und Sud in Amerika. Gemeinf

scher Hine

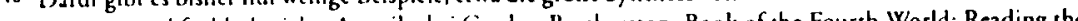
sein im vor-und frutikolomalen Amerika bei Gordon Brotherston, Book of the Foun World. Reading the Nacive Americas through their Lierature (Cambridge 1922), oder das weigehend auf ind hnischen Quellen basserende Werk von James Lockharr, The Nahuas after the Conquest: A Social and Cultural History of the Indians of Central Mexico. 16th through 18th Cencuries (Stanford, Cal. 1992). Für Asien etwa: George Elison, Deus Destroyed: The Image of Christianity in Early Modern Japan (Cambridge, Mass./London 1973). re Erklärungsmodelle (Sozialimperialismus“, „unruhige Grenze“, „Kollaboration“, usw.) bereithält. Ähnliches fehlt für das, was man pauschal und vorläufig als „kulcurellen Imperialismus “ bezeichnen muß.17 Soll ein Rückfall in eine bloß illustrierende, ihr bildkräftiges Material stoffhuberisch auf den bunten Effekt hin arrangierende Kulturgeschichtsschreibung vermieden werden, so sind umfassende und kritisch auswählende Sichtungen des Theorieangebotes, wie es vornehmlich von Kulturanthropologie und Soziologie, daneben neuerdings auch von der Literaturwissenschaft bereitgestellt wird, unerläßlich. ${ }^{18}$

Angesichts dieser Schwierigkeiten hat ein kulturgeschichtliches Interesse an der europäischen Expansion zunächst den Weg der Untersuchung europäischer Perzeptionen außereuropäischer Gesellschaften genommen. Die ungeheure Fülle überlieferter Äußerungen von Europäern über außerokzidentale Gesellschaften in Reiseberichten sowie in deren Anthologisierungen und sekundären Verwertungen, in Akren von Diplomatie und Kolonialregierungen, dazu eị reiches Bildmaterial haben hier einen fruchtbaren Forschungszweig eröfnet. Allerdings stehen Untersuchungen dieser Art in einem schwer auflösbaren Dilemma. Suchen sie, auf dem Wege über die europäischen Quellen zu einem Verständnis realer interkultureller Kontaktsituationenen vorzudringen, so verstricken sie sich, wenn keine unabhängigen Zeugnisse der ,anderen“ Seite benutzt werden oder wenn man nicht skrupelhafteste textkritische Vorsicht walten läßt, ${ }^{19}$ zirkulär in den Befangenheiten der zeitgenössischen europäischen Beobachtungsquellen. Zieht man jedoch, wie einige Autoren des "new historicism“, aus dieser Schwierigkeit den Schluß, europaiische Texte über Außereuropäisches überhaupt nicht mehr auf ihren möglichen Sachgehalt hin zu lesen, sie also ausschließlich als Spiegelkabinett okzidentaler Vorurteile und Phantasmagorien aufzufassen, als eine Sonderform von „fiction“, dann verschwindet die Realität des Kulturkontakts hinter dem Vorhang ihrer textlichen Verschleierung. Was in der Tendenz der wertenden Aussage zu einer ungemilderten Verurteilung europäischer Verblendung führt, resultiert methodisch dann häufig in nichts als deren Verdoppelung: einem Narzißmus, der nicht länger nach der Wirklichkeitsadäquarheit europäischer Fremdbilder und nach ihren 17 Die unter diesem Schlagwort geführten Diskussionen lassen of historischen Tiefgang vermissen. Ein
Beispicl dafür ist John Tomlinson, Cultural Imperialism (London 1991). Anregender ist etwa der Versuch Beispiel dafür ist John Tomlinson, Cultural Imperialism (London 1991). Anregender ist etwa der Versuch des philippinischen Historikers Renato Constantino, die sozialökonomische Dependenztheorie auf den Bereich der Kultur zu übertragen. $V_{g}$ l. R. Constant

Essays on Cultural Decolonization (London 1978). is Vgl. die Unersuchungen zu kolonialen Diskursen (oben Anm. 14); Dietrich Krusche, Literatur und Fremden. Erkundung kultureller Grenzen in Literatur und Publizistik (Frankfurt a. M. 1994). Ein anregender Beitre aus philosophiegeschichelicher Siche ist Hinsich Fïnk-Eirel. Die Philosophie und die Wilden. der Beitrag aus philosophieger die Bederung des Furopäische Geistesgeschichee (Hamburg 1994). Hilfreich sind Ober die Bedeurung des fres der bisher allerdings kaum historisch interessierten "Xenolngie", vgl. Alois auch manche Uberlegungen der bisher allerd ges kefiffe und Problenfelder der kulturwissenschaftlichen Wierlacher (Hrsg.), Kulturthema Frem

19 Vgl. Adam Jones, Zur Quellenproblematik der Geschichee Westafrikas 1450-1900 (Stuttgart 1990) 30ff. 
realen Folgen fragt, sondern nur noch nach ihren innertextuellen Generierungssregeln und ihrem ideologischen Gehalt. Selbst wenn eine solche ,reduction of experience to the meanings that shape it "20 mit der Brillanz ihrer virtuosesten Praktiker geschieht, läßt sich eine Kulturgeschichte der europäisch-außereuropäischen Begegnung darauf schwerlich aufbauen.

Die historische Untersuchung tatsächlicher Kontakte zwischen Europäern und Nicht-Europäern unter dem Gesichtspunkt ihrer kulturellen Voraussetzungen und Konsequenzen bedarf anderer konzeptioneller Hilfen, als der „new historicism“ sie anbieten kann. Ein bewährter Ausgangspunkt ist die Taxonomie von Formen der „Kulturbegegnung“, die Urs Bitterli vorgeschlagen hat. ${ }^{21}$ Bitterli unterscheidet 1) die "Kulcurberührung“, d.h. den punktuellen Erstkontakt, „das in seiner Dauer begrenzte, erstmalige oder mit großen Unterbrechungen erfolgende Zusammentreffen einer Gruppe von Europäern mit Vertretern einer überseeischen Kultur";22 2) den aus der friedlichen Kulturberührung oft durch unmittelbare oder von den Einheimischen (vermeintlich) provozierte Gewaltanwendung der Europäer hervorgehenden „Kulturzusammenstoß"; als relativ gewaltfreien Dauerzustand 3) die "Kulturbeziehung“, also „ein dauerndes Verhältnis wechselseitiger Kontakte auf der Basis eines machtpolitischen Gleichgewichts oder einer Patt-Situation"; ${ }^{23}$ schließlich 4) als selten erreichten irenischen Extremzustand die aus Akkulturationsprozessen hervorgehende „Kulturverflechtung". ${ }^{24}$ Bitterli benulzı, ohne größere theoretische Ambitionen daran zu knüpfen, diese Klassifikation von Kontakttypen, um ein weltweit gesammeltes, zumeist aus einer naiven, also auf die Raffinessen von „Diskursanalyse“ und dekonstruktivistischer Literaturwissenschaft verzichtenden Lektüre von Reiseliteratur geschöpftes Belegmaterial zu ordnen. Innerhalb dieses Rasters lassen sich dann die Geschichten einzelner Kulturbegegnungen erzählen; den Chancen einer durchkomponierten Gesamtgeschichte der europäischen Expansion muß Bitrerli von seiner typisierenden Vorgehensweise her skeptisch gegenüberstehen, wenngleich aus anderen Gründen, als die Kritiker von "master narratives" sie vorbringen. Seine Perspektive ist bewußt europazentrisch; Kulturkontakte werden vor dem Hintergrund der europäischen „Geistesgeschichte" betrachtet.

20 John E. Toews, Incellectual History after the Linguistic Turn: The Autonomy of Meaning and the Irreducibility of Experience, in: American Historical Review 92 (1987) 879-907, hier 906. Ein neueres Beispiel dafiir wäre Stephen Greenblate, Marvelous Possessions: The Wonder of the New World (Oxford Beispiel di).

21 Urs Bitterli, Die „Wilden" und die „Zivilisiereer”. Grundzüge einer Geistes- und Kulturgeschichre der 21 Urs Bitterli, Die "Wilden" und die "Zivilisiereen". Grundzüge einer Geistes- und Kulturgeschichre der
curopaisch-übersecischen Begegnung (München 1976) 81 Iff.; ders., Alte Welt - Neue Welt. Formen des curopaisch-ubersecischen Kulturkoncaktes vom 15. bis zum 18. Jahrhundert (München 1986) 17-54; ders., Kulturlocgrgnung und Kuleurzusammenstoß in der Siche des Kolonialhistorikers, in: Alfred Wendehorst/]ürgen Scluarider (Hirsy.). Begegnungsraume von Kulturen (Ncustade a. d. Aisch 1982) 75-88.

22 Bitserli. Alte Welt (wie Aum. 21) 17

2 Lbd. 42.

24 Bitcerli, Die "Wilden" (wic Anm. 21) 161-173.
Bitterlis einfache und einleuchtende Taxonomie, die im Hinblick auf die Epoche zwischen Kolumbus und Cook entwickelt wurde und deren Brauchbarkeit für spätere Phasen stabiler Kolonialverhältnisse noch nicht geprüft worden ist, unterscheidet Grundformen des Kulturkontakts nach den beiden Kriterien von Dauer und Gewaltsamkeit; die Skala reicht vom genozidalen Überfall bis zur dauerhaften Symbiose der Kulturen. Die Trennung zwischen Europa und Nicht-Europa, die den Rahmen aller erfaßten Kontaktsituationen bildet, wird dabei als unproblematisch vorausgesetzt; die Abgrenzung zwischen Europäern und den "Anderen" scheint den Zeitgenossen wie auch dem Historiker unmittelbar evident zu sein; der Kontakt findet statt zwischen den Angehörigen deutlich identifizierbarer zivilisatorischer Einheiten. Gerade dies jedoch müßte überprüft werden. Differenzen und Distanzen zwischen Kulturen sind historisch variable Größen, Konstrukte wechselnder Selbst- und Fremdzuschreibungen. Auf beiden Seiten des interkulturellen Zusammentreffens kommt es zu Prozessen von Identitätsveränderung und Gruppenbildung, von Abgrenzung und Annäherung. Imaginative Entwürfe vom Anderen - „Bilder“" keinesfalls im schlichten Sinne von Abbildungen - unterliegen (was auch Bitterli anerkennt) keinem langfristigen Entwicklungsgesetz der allmählichen mentalen Annäherung zwischen Kulturen im Zuge der Herausbildung eines kommunikativen Globalzusammenhangs. Mit zunehmendem Wissen voneinander kann die Verständnislosigkeit wachsen. Kulturelle Unterschiede, darauf hat Fredrik Barth in einem einflußreichen Aufsatz hingewiesen, können durchaus trotz intensiver interkultureller Beziehungen und Abhängigkeiten dauerhaft fortbestehen. ${ }^{25}$ „Kulturbeziehung“ kann leicht zum „Kulturzusammenstoß" regredieren. "Bilder" vom jeweils Anderen formieren und verändern sich in einem engen Wechselverhältnis mit den tatsächlichen Ungangsweisen. Perzeptionen und Interaktionen bedingen einander auf eine Weise, die nicht in lineare Kausalitätsrelationen aufzulösen ist. Zum Spektrum der Möglichkeiten gehörten sowohl die Stabilisierung und lernpathologische Radikalisierung anfänglicher "Mißverständnisse“ im Sinne von "self-fulfilling prophecies", die Umsetzung also von Differenzwahrnehmungen in Dif ferenzierungspraktiken, als auch umgekehrt die nachträgliche Ideologisierung konkreter Macht- und Herrschaftsverhältnisse; beides läßt sich etwa an den verschiedenen Ausprägungen von Rassismus beobachten.

Urs Bitterlis Typologie von Kulturbegegnungen im Zuge der neuzeitlichen Expansion Europas kann in dreifacher Weise weitergedache werden: in Richtung auf 1) eine Verfeinerung der Mischformen und Übergänge zwischen den Grundrypen, 2) die historisierende Problematisierung von Ver- und Entfremdungsprozessen zwischen Kulturen und 3) die flexiblere Zuordnung zueinander von Perzeptionen und Praktiken. Als theoretischer Fokus kann dabei das Konzept der „kulturellen Grenze“ dienen. ${ }^{26}$

25 Fredrik Barth, Introduction, in: ders. (Hrsg.), Ethnic Groups and Boundaries: The Social Organization of Culrural Difference (Bergen/Osto/London 1969) 9-38, hier 10.

26 Zum Teil werden hier Überlegungen aus einem früheren Beitrag weitergeführr: Jürgen Osterhammel, Distanzerfahrung. Darstellungsweisen des Fremden im 18. Jahrhundert, in: Hans-Joachim König/Wolfgang 


\section{Drei Begriffe von "Grenze“}

Die Vorstellung von "Grenzen“ - mit dem semantischen Feld Begrenzung, Entgrenzung, Grenzüberschreitung - gehört zu den schillerndsten Metaphern-Konzepten in den heutigen Sozial- und Kulturwissenschaften, zugleich zu denen, von welchen man sich neuerdings zusammenfassende Orientierungen in der Gegenwart zu erhoffen scheint. ${ }^{2 \prime}$ Als Begriff ist sie teils der Philosophie, teils der Geographie entlehnt worden. Georg Simmel, der wiederholt auf die „Raumbedeutung der Dinge und Vorgänge“ hinwies, ${ }^{28}$ überserze die Vorstellung räumlicher Begrenzung in die Idee der ,soziologischen Grenze“ und postulierte: „Die Grenze ist nicht eine räumliche Tatsache mit soziologischen Wirkungen, sondern eine soziologische Tatsache, die sich räumlich formt. " ${ }^{29}$ Die Systemtheorie, insbesondere bei Niklas Luhmann, siehr die Bestimmung von Grenzen als "das wichtigste Erfordernis der Ausdifferenzierung von Systemen “. ${ }^{30}$ Eine solche Grenzbestimmung sei nicht allein eine analytische Leistung des unterscheidenden Betrachters, sondern werde bereits von den Systemen selbst geleistet. Der Ethnologe Fredrik Barth siehr in „boundary maintenance" die wichtigste Anpassungsstrategie einer ethnischen Gruppe; charakteristisch für eine solche sei ihr Grenzverhalten, niche so sehr ihre durch konstante Merkmale beschreibbare kulturelle Substanz. ${ }^{3}$

Es ist wichtig, sich solche allgemeinen und abstrakten Bestimmungsmöglichkeiten von "Grenze“" zu vergegenwärtigen, wenn man nach den spezielleren Verwendungen des Begriffs als geschichtswessensshafflicher Kategorie fragt. Eine hinreichend plastische Vorstellung von kulturellen Grenzen kann nur so entwickelt werden, daß sich Verbindungen zeigen lassen zwischen der Metaphorik von kultureller Differenz, Fremdhei und „orherness" und jenen konkreten und sichtbaren Interaktionssträngen, als welche Historiker seit jeher Grenzen verstanden haben. Vor allem drei Grenzformen sind dabei zu unterscheiden - auch wenn sich in der Fülle historischer Situationen reine Ausprägungen dieser Typen selbstverständlich nur ausnahmsweise finden: die imperiale „Barbarengrenze", die nationalstaatliche Territorialgrenze und die Erschließungsgrenze. ${ }^{32}$

Reinhard/Reinhard Wendr (Hrsg.), Der curopäische Betrachter außereuropäischer Kulturen. Zur Problematik der Wirklichkeitswahrnehmung (Berlin 1989) 9-42.

27 Der Weltkongreß für Soziologie im Juli 1994 in Bielefeld stand unter dem Motro „Umserittene Grenzen und wechselnde Solidaritätert".

${ }_{28}$ Georg Simmel, Suziologic. Untersuchungen über die Formen der Vergesellschaftung [1908], hrsg. von Otthein Rammstedt (= Georg Simmel Gesamtausgabe, Bd. I1) (Frankfurt a. M. 1992) 689.

29 Ebd. 697

30 Niklas Luhmann, Soziale Systeme. Grundriß einer allgemeinen Theorie (Frankfurt a.M. 1984) 54, 177 f.

31 Barth (wie Anm 25) 14f. Zum Diskussionskontext vgl. Richard Jenkins, Social Anthropological Models of Incer of Inter-erhnie Relations, m: John Rex/78. Weitere wichuige theorecische Beiträge zu Grenze und Abgren-

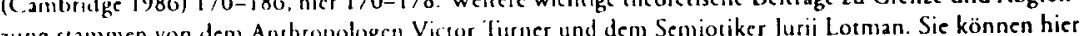
inche erorerer werden

12 Andere, wesentlich kompliziercere Typologien sind vorgeschlagen worden, so elwa in einer noch immer anregenden Alhandlung von Siephen B. Jones, Boundary Concepes in the Setting of Place and Time, in: Annals of the Associacion of Amcrican Geographers 49 (1959) 241-25s.
Die imperiale „Barbarengrenze“, wie sie sich an der Peripherie des Imperium Romanum von Nordeuropa bis Afrika, an der durch die Große Mauer gesicherten und visualisierten Nahtstelle zwischen chinesischer Ackerbau- und innerasiatischer Hirtenkultur, in Gestalt der habsburgischen Militärgrenze gegenüber dem osmanischen Reich ${ }^{33}$ oder am nordwestlichen Rand Britisch-Indiens (der „North-West frontier“ vis-ג̇-vis Afghanistan) findet, ${ }^{34}$ ist eine defensive Sicherheitszone, durch welche sich Imperien am Punkt ihrer maximalen Ausdehnung gegen zumeist tribal organisierte, eine hochmobile Kriegführung praktizierende Nachbarvölker zur Wehr setzen. Diese Grenze kann über längere Zeiträume statisch sein, muß also nicht als Sprungbrett zu weireren Eroberungen dienen. Sie ist oft durch aufwendige militärische Grenzanlagen vom linearen „Limes"-Typ charakterisiert, denen, unabhängig von ihrer tatsächlichen Wirksamkeit, eine hohe symbolische Bedeutung als Trennungslinie zwischen imperialer, kosmisch geordneter „Zivilisation“ und frei schweifendem, anarchischem „Barbarentum" zugemessen wird. ${ }^{35}$ Trotz ihrer spektakulären Undurchdringlichkeitssymbolik ist die imperiale „Barbarengrenze“ in der Praxis meist durchlässig für „kleinen Grenzverkehr" aller Art; ohnehin staffelt sie sich diesseits sichtbarer Mauern, Walle und Palisaden in die Tiefe eines bikulturell durchdrungenen Hinterlandes. In Phasen, die auf imperiale Expansion und erste Grenzsicherung folgen, kann es zum wichtigsten Zweck linearer Barrieren werden, die „Barbaren“ jenseits der Grenze von denjenigen auf imperialem Gebiet zu trennen, die im Begriffe sind, sich kulturell zu romanisieren, zu sinisieren, usw. ${ }^{36}$ Schon für die römische Republik ist bemerkt worden, „daß die Römer eher versuchren, die Einheimischen in Römer zu verwandeln, als ihre eigenen Leure in verlassene Landstrichen zu bringen" ${ }^{37}$ Ähnliches ließe sich für China sagen. Oft dienen Orte entlang der Grenze als diplomatische Stützpunkte für ein „Management" militärisch nicht dauerhaft pazifizierbarer tribaler Nachbarn, die als Klienten in Reziprozitätsverhältnisse eingebunden werden; Ziel solcher Strategie oder Takrik ist

33 Die Militärgrenze wurde von österreichischer Seire vielfach als „Barbarengrenze“ interpretierr, war aber tatš̈chlich ein Verteidigungsgürrel gegenüber einem hochorganisierten Nachbarimperium. Auf dem Höhepunkt ihres Ausbaus, in der zweiten Hälfre des 18. Jahrhunderts, zog sie sich als 20-60 Meilen breite Zone puber ausend Kilometer von der Adria bis zum nördlichen Ende der Moldaukarpaten hin. Vgl. Gunther $E$ Rothenberg. Die österreichische Milië̈rgrenze in Kroatien 1522 bis 1881 (Wien/München 1970) 9-14; Jean Nouzilic, Histoire des frontieres: L'Autriche ar l'Empire ottoman (Paris 1991) bes. $57 \mathrm{ff}$.

34 Die North-West Frontier was allerdings niche mir durchgehenden Verteidigungsanlagen befestigt. Sie war 34 Die North-West Frontier war allerdings niche mir durtageheren ,a zone or belt of mountainous country of varying width, stretching over a distance of abour 1,200 miles 1707-1947 (Delhi 1985) 525. 1707-1947 (Delhi 1985) 525.
35 Die - je nach Zuschreibung wechselnde - symbolische Bedeutung der Grenzbauwerke betont am chinesischen Beispicl Arthur Waldron. The Great Wall of China: From History to Myh (Cambridge 1990). Auf der Grundlage von Waldrons wichtigem Buch: Gudula Linck, Die Chinesische Maucr: Realität und Mythos einer steinernen Grenze, in: SOWI 20 (1991) 164-171. Vgl. auch Thomas I. Barfield, The Perilous Frontier: Nomadic Empires and China (Oxford 1989) bes. 5-8.

36 Edward N. Lutwak. The Grand Strategy of the Roman Empire: From the First Censury A. D. to the Third (Baltimore/London 1976) 78.

37 Stephen L. Dyson. The Creation of the Roman Frontier (Princeton, N. J. 1985) 
of die Transformation von militärischem Gleichgewicht in eine durch kulturelle Bindungen untermauerte politische Abhängigkeit. Die imperiale „Barbarengrenze“ ist jüngst als geopolitische Leitmetapher zum Verständnis der Weltlage nach dem Ende des Ost-West-Konflikts wiederbelebt worden: der reiche Norden repräsentiere das neue, sich vom Elend des Südens abschotrende und sich zugleich normativ auf einen halbierten Universalismus zurückziehende Imperium. ${ }^{38}$

Die nationalstaatliche Territorialgrenze ist demgegenüber eine Demarkationslinie zwischen zwei im Prinzip ähnlich organisierten politischen Gebilden. Grenzen sind in diesem Verständnis nicht Distanzierungszonen zwischen Imperien und ihren Umwelren, sondern Staatsgrenzen, ${ }^{39}$ genauer: durch Hoheitssymbole und Organe staatlicher Machtpräsenz markierte Linien zwischen territorialen politischen Verbänden, oder noch anders: Maximalpunkte durchsetzbarer Jurisdiktions- und Souveränitätsansprüche - spätestens seit dem 19.Jahrhundert in der Regel von Nationalstaaten. 40 Ihre Festlegung erfolgt als Kodifizierung der Ergebnisse von Wanderung, Krieg und Staatsbildung meist durch rechtsförmige Übereinkünfte zwischen Regierungen. Schon der Geograph Friedrich Ratzel machte jedoch darauf aufmerksam, daß neuzeitliche politische Grenzen trozz mitunter früher vertraglicher Festlegung sich vielfach erst allmähzisieren. ${ }^{41}$ Ratzel betonte auch die vielfäligen Funktionen der Grenze als "peripherischem Organ" eines zentralisierten Staatsgebildes: „Durch diese Funktionen wird die Grenze zu einem höchst eigentümlichen Organ des Gebietes, das von ihr umschlossen wird, und nimmt zu seinen anderen Teilen eine Stellung ein, die sich durchaus nicht in der Vorstellung erschöpft, daß in ihr die äußersten Punkte des Gebietes gelegen seien." ${ }^{2}$ Der Grenze wird damit als randständigem Raum trotz des Bezugs auf domi-

Jean-Christuphe Rufin, L'Empire at les nouveaux barbares: Rupture Nord-Sud (Paris 1991) (225 über

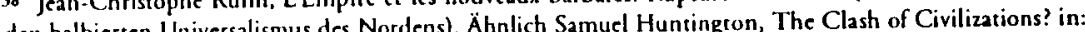
dent halbierten Univerallon Foreign Affairs 72.3 (19) 22 a. M. 1993) 56 . Eine weniger pessimistische geopolische Vision erwa be Max

The Real World Order: Zones of Peace - Zones of Turmoil (Chathe Neurschlands 39 Diesen Gremzbegriff verwenden die meisten Beiträge in Alexander Demandr (Hirs.), Deutschlands Grenzen in der Geschichie (München 21991). Eine weltweite historische Obersicht über den „decoupage du monde" gibr Michel Foucher, Fronts er frontières: Un tour du monde geopolitique (nouvelle ed. Paris 1991) 99-134; daneben immer noch Paul Guichonnct/Claude Raffestin, Géographie des frontières (Paris 1974) 82-145, sowie das klassische Werk eines Grenzen ziehenden Praktikers: Sir Thomas Holdich, Political Frontiers and Boundary Making (London 1916)

to Grundlegend jezzr Perer Sahlins. Boundaries: The Making of France and Spain in the Pyrenees (Berkeley/Los Angeles/Oxford 1989); ders., Natural Fronciers Revisited: France's Boundaries since the Seventeenth

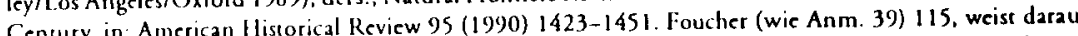
hin hin. daß Z Zeriler des In dien-Bume

dien-Burna) 2ur 4.

auch J. R. V. Prescutt, Linfulin
42 Raczel (wie Anm. 41) 510. nierende Zentralstaatlichkeir ein gewisses Eigenleben als Folge „peripherischer Auf lockerung " 43 zugesprochen. Of sind es solche schwächer kontrollierten Zwischengürtel, an denen sich politische und echnische Neubildungen ergeben und mitunter zu Herausforderungen für die beiderseitigen Zentren entwickeln. In Auseinandersetzung mit Ratzel und seinen Nachfolgern in der deurschen politischen Geographie und Geopolitik haben besonders Lucien Febvre und die französischen Geographen Albert Demangeon und Jacques Ancel ein umfassendes kulturhistorisches Konzept der zwischenstaatlichen Grenze entwickelt. ${ }^{44}$ Neuere Beiträge betonen die Ambivalenzen in Begriff und Wirklichkeit zwischenstaatlicher Grenzen (,borders“, „boundaries“), deren jeweilige Geschichte aufgefaßt werden könne als „ein komplexes Wechselspiel zwischen zwei Grenzkonzepten - zonal und linear - und zwei Ideen von Souveränität zwischen zwei Grenzkonzepten - 20 al und territorial."45 Grenzen haben zur Formierung von Identitäten und Gegenidentitäten Anlaß gegeben; sie sind zuweilen zu Kristallationssträngen für besondere Grenzgesellschaften geworden. ${ }^{46}$

Die Erschließsungsgrenze oder "frontier" ist die Expansionsgrenze par excellence. Sie ist in der Regel eine agrarische Siedlungsgrenze, ${ }^{47}$ kann aber etwa auch die Form einer Grenze der bergbaulichen Ressourcenerschließung annehmen oder etwa als ,commercial frontier " 48 oder Urbanisierungsgrenze verstanden werden. ${ }^{49}$ Sie ist, anders als die

\section{Ebd. 513 .}

44 Auf die Bedeurung der französischen Beirräge weist Hans Medick in seinem wichrigen Themenaufriß hin: Zur politischen Sozialgeschichice der Grenzen in der Neuzeir Luropas, in: SOWI 20 (1991) 157-163, bes. 158f Das Pionierwerk war Albert Demangeon/Lucien Febvre. Le Khin: Problemes d'hiscoire er d'eco-

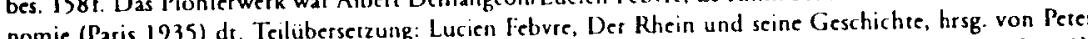
nomie (Phörter (Frankfure a. Mew York 1994); von Febvre auch. Frontière" - Wort und Bedeutung [1928] Schorter (Frankfurr a. M. Nes Historikers, hiss von Ulrich Raulff (Berlin 1988) 27-37. Bedeutend ist auch in: ders., Das Gewissen des Historikers, hrsg. von Uf verwendende Werk von Jacques Ancel, Géographic des das typologisch angelcre, einen weiren $\mathrm{Crn}$. frontitres (3e ed. Paris 1938): cin $\mathrm{Ce}$

politischen Bedeutung (Berlin 1927).

45 Sahlins, Boundaries (wic Anm. 40) 7. 46 Leider gibe es noch keine neuzeitliche Entsprechung solch neuer me Robert Bartlett/Angus Mackay (HisB Anthony Tuck (Hrsg.), War and Border Sociecies in the Middle Ages (London 1992) bes. über die angloschottische Grenze.

17. Dies ist die hauptsächliche Begriffsverwendung in der Kulturgeographie. Vgl. etwa Hans-Jürgen Nitz, Landerschließung und Kulturlandschaftswandel an den Siedlungsgrenzen der Erde: Wege und Themen de Forschung, in: Götringer Geographische Abhandlungen 66 (1976) 11-24.

Forschung. in: Gottinger Geographische Abhischen 48 Dic ,trading frontier" ist seit Turners klassischem Aufsatz of als Merkmal der franzosischen Kolonisation in Nordamerika verstanden worden, in Gerer in American History [1893], in: ders.. The Frontier in American Turner, The Signiffence of he Fen History (newed. Tucson/London 1986) 1-38, hier 13f. Heute wird der Begrif weit bei Ralph Austen, African amerikanischen Bezugsberrich hinaus verwender. Exezal Dependency (London/Portsmouch, N. H. 1987)

Economic

Vl. etwa David Hamer, New Towns in the New World: Images and Perceptions of the Nineteenth-Century Urban Frontier (New York 1990). Manch andere Arten von "f frontiers" sind ausfindig gemache word 
im Grunde defensive und stationäre imperiale „Barbarengrenze“, in ständiger Bewegung, eine „wandernde Grenze“. so Die Expausion kann durch staatliche Instanzen gesteuert oder gefördert werden, etwa mittels rechtlicher Garantien für den Landerwerb und in extremen Fällen sogar durch Zwangsansiedlung. Oft wird sie militärisch abgesichert - bis zum Ende des 19. Jahrhunderts war der Kampf an der Indianergrenze die wichrigste reguläre Aufgabe der Armee der USA. In der Regel geht die eigentliche Erschließungs- und Kolonisierungsdynamik aber , anarchisch" von privaten Individuen und Gruppen aus. Im Gegensatz zum Imperium, das gegenüber den externen „Barbaren“ das Recht der Eroberung für sich in Anspruch nimmt und sich oft auch selbst zivilisatorische Missionsaufträge erteilt, beruft sich eine landnehmende Siedlerschaft auf die naturalistische Vorstellung, „unberührte Wildnis"st durch Arbeit zurückzudrängen und sich damit einen Rechtstitel auf vorgeblich herrenloses Land, terra nullius, zu erwerben. ${ }^{22}$ Die Existenz von Rechten der Ureinwohner wird bestritten, ihre "Zivilisierung" of für aussichtslos gehalten. ${ }^{53}$ Die Erschließungsgrenze als bewegliche "frontier" im engeren Sinne ist noch weniger als dic beiden anderen Grenztypen eine deutliche Demarkationslinie. In der neueren Grenzforschung wird sie betrachtet „nicht als Grenzlinie, sondern als ein Territorium oder eine Zone gegenseitiger Durchdringung von zwei zuvor getrennten Gesellschaften". ${ }^{\text {s4 }} \mathrm{Zu}$ einer Frontier-Situation gehören drei Elemente: „ein Territorum. zwei oder mehr ursprünglich voneinander unabhängige Völker und der Prozeß, durch den Beziehungen zwischen den Völkern innerhalb des Territoriums entstehen, sich entwickeln und schließlich zu Kristallisa-

so Dierrich Gerhard, Neusiedlung und institurionelles Erbe. Zum Problem von Turners „Frontier”, eine vergleichende Geschichrsbetracheung [1957], in: ders., Alte und neue Welt in vergleichender Geschichrsbevergleichende Geschichtsbetracheung [1957], in: ders., Alte und neue Welt in vergleichender Geschichrsbe-
erachtung (Göttingen 1962) 108-1 40, Zirat 108. Dieser Aufsarz bleibe einer der anregendsten Beiträge zum

Phänomen der "frontier". 51 Daß er keine Spuren in der Landschaft hinterlasse, ist ein alter europäischer Topos bei der Identifikation
des "Wilden". Er findet sich schon früh bei der Beurteilung der Indianer. Vgl. erwa Bernard Sheehan, des "Wilden". Er finder sich schon früh bei der Beurteilung der Indianer. Vgl. erwa Ber
Savagism and Civility: Indians and Englishmen in Colonial Virginia (Cambridge 1980) 68 .

\$2 Vgl. Jörg Fisch, Der Mythos vom leeren Land in Sudafrika oder Die verspärere Entdeckung der Afrikaner durch die Afrikaaner, in: Heinz Duchardt/Jürg A. Schlumberger/Peter Segl (Hrsg.), Afrika: Entdeckung und Erforschung eines Kontinents (Köln/Wien 1989) 143-164. Wie zäh sich der Mythos von der unbewohnten Wildnis des vor- und frühkolonialen Amerika bis heute in der Geschichesschreibung der USA behauptet, ce zeigr james H. Mer 46 (1989) 94-119, hier 98 f 1010.112

¿3 Dies gilt besonders deutlich für Nordamerika bis etwa zum Bürgerkrieg. - Eine solch brutaie Naturali"Dies gilt besonders deutlich für Nordamerika bis etwa zum Bügerkieg. sierung von Zivilisationsdifferenzen hat vor dem 20. Jahrhunderr an der osilichen frome Europas selten eine größere Rolle gespielt. Die vielleicht bekannteste neuere Interpretation des Phanomens der „Ostkolonisation" (in einem sehr weiten Sinne) hat vielmehr gerade die kulturelle Transformation Osteu ropas unter-

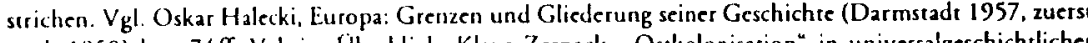
engl. 1950) bes. $74 \mathrm{fr} . \mathrm{Vgl}$ im Uberblick: Klaus Zernack, .Ostkolonisation“ in universalgeschichtliche Perspektive, in: Gangolf Hubinger/Jurgen Osterhammel/Erich Pelzer (Hrsg.). Universalgeschichte und Nationalgeschichren (Freiburg i. Br. 1994) 105-116. Das Problem der kulturellen Grenze im Osten und Südosten Europas wird im folgenden niche vertieft werden.

34 Howard Lamar/Leonard Thompson, Comparative Fronticr History, in: dies. (Hrsg.), The Frontier in History: North America and Southern Africa Compared (Ncw Haven 1981) 31-13, hier tionen führen".5s "Frontiers", so heißt es in ähnlicher Tendenz in David J. Webers vorbildlicher Darstellung der spanischen Grenze in Nordamerika, seien aufzufassen als Zonen der Interaktion zwischen zwei unterschiedlichen Kulturen, als Orte, wo die Kultur der Invasoren und die ihrer Opfer [the invaded] mitcinander ringen und im Rahmen ihrer physischen Umwelt eine nach Zeit und Ort spezifische Dynamik hervorbringen". ${ }^{6}$ Im Gegensatz zu einer solch doppelseitigen, anthropologisch interessierten Grenzauffassung, die voraussetzt, daß eine wandernde Grenze unweigerlich auf die cigene Grenze einer anderen Zivilisation stoßen wird, nahm die ältere Geschichtsschreibung in der Nachfolge Frederick Jackson Turners ausschließlich die Perspektive der siegreichen Weißen ein. Turner sah die amerikanische Frontier lakonisch als "the meeting point between savagery and civilization" und als tief in die "Wildnis" ausstrahlende Zerstörerin der "primitiven indianischen Lebensweise“. 57 Ihn interessierte nicht die zwischenkulturelle Grenzgesellschaft, sondern die weiße Grenzergesellschaft, in der sich, die gesamte Nation beeinflussend, die Eigentümlichkeiten Amerikas ausprägten. ${ }^{58}$

Die Turnersche Vorstellung von der voranrückenden Pioniergrenze ist zur vielleicht bildkräftigsten Metapher der europäischen Welteroberung geworden. Walter Prescott Webb, einer der frühesten umwelthistorischen Interpreten der amerikanischen Frontier, gab dem Konzept der „Great Frontier" eine universale Bedeutung und erklärte das neuzeitliche Westeuropa zur globalen Metropolis, die ihre Grenzen in Übersee suche. Diese Denkfigur übernahm der Soziologe Immanuel Wallerstein in den 1970er Jahren in seine historische Theorie des "modernen Weltsystems". Die wichtigste Funktion der Außengrenze dieses zyklisch expandierenden Systems besteht, Wallerstein zufolge, in der "Inkorporation" bisher unberührter Zonen in die globale Arbeitsteilung. Eine solche Einverleibung ist nicht nur mit einer sozialökonomischen Umwälzung in den erfaßten Gebieten verbunden, sondern auch mit dem Umbau ihrer staatlichen Struk-

ss Ebd.

36 David J. Weber, The Spanish Frontier in North America (New Haven/London 1992) 11

37 Turner (wie Anm. 48) 3.13. Eine besonders cinflußreiche Formulierung Turnerscher Orthodoxie ist Ray Allen Billington, Westward Expansion: A History of the American Frontier (New York 51982).

so Die Kritik an Turner serze allerdings schon bald nach scinem Tod in Jahre 1932 ein. Die heutige Forschung zur amerikanischen Frontier - vgl. als neueste Synthese: Clyde A. Milner II/Carol A. O'Conor/Martha A. Sandwciss (Hrsg.). The Oxford History of the American West (New York/Oxford 1994) baur vielfach af solchen frühen Alternativen zur Turner-These auf. Vgl. John Mack Faragher, The Frontier Trail: Rethinking Turner and Reimagining the American West, in: American Historical Review 98 (1993) 106-117, hier 109. Zur Turner-Diskussion vol. Charles S. Pererson, Speaking for the Past, in: Mil106-117, hier 15 Zn 743-769, sowie die krivischen originell weiterfürenden Übersichren bei Donald net/O'Connor/Sandweiss, 743-769, sowie die kritischen, originell Wecerthenden Ubersichen bei Donald Worster New Wes, Tre We

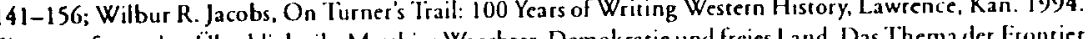
Einen umfassenden Überblick gite Marthias Waechrer, Demokratie und frcies Land. Das Thema der Frontier
in der amerikanischen Geschicheswissenschafr (phil. Diss. Freiburg i. Br. 1993).

\$9 Walter Prescort Webb, The Great Fronticr (Austin, Tex. 1951) $7 \mathrm{f}$ 
in der Regel der Aufwingung kolonialer Herrschaft. ${ }^{\circ}$ Deuticher als Wallerstein in Universalhistoriker der Gegenwart, Wilhat schließlich der führende amerikanische Univer weltweiten "Great Frontier" in die liam H. McNeill, seine Oberiegull ${ }^{61}$ Er definierr die Erschließungsgrenze insofern enger als Wallerstein, als er darunter nicht jede Eingersteht, sondern nur die Ausdehdas von Europa geformte kapitalistische Wün besiedeltes Land zum Zwecke seiner wie sie nung europäischer Kontrolle uber dun Formen von Arbeitszwang nach sich, wie telbaren Nurzung; dies zog in der Regel Formen vönger vorkamen. ${ }^{62} \mathrm{McNeill}$ geht inde in der europäischen Metropolis selbst nich läger da Rande bedenkt. Die Erschließungsgrenze sei eine Front kulturelle Charakter dest am Rande bedertureller Zerstörung gewesen. Der "drastisch einceice" 63 sei um die sungegnung, der entlang der Great Fronter Offensive der okzidentaMitre des 20. Jahrhunderts zu seinem Ende gekommen. Der Ontgegen.

\section{Nulsurelle Giremism}

Det konkrereste Cehslt des Begritts der kuluurellen Circine ist dergenige der Sprach renze. Nur die raumlutie Verteilung von Sprativerwendung kamn einigermaßso grenze. Nir die and auf Karten festgehalien werden. Sehond de Cieographie der Reliexakt ermittelt und auf Karten festgen, dabei eines der wichtigsten bei der Ausfullung gionen ist ein heikler des Konzepts der kulturellen Gr", die die pangermanische Geopolitik der Zwischenurrenzen" des „Deutschtums", die die pangercher Kritiker bemerkt denn auch, die kriegszeit entwarf. Deren schärfster zeitgenossischer Krie Spiritualisierung von Grendeutschen Geographen seien mit ihrem Bemühen um die Spiritua " gewesen. ${ }^{64}$ Der im Munst der Kartographie des Ungreilbaren gewesen solche fläfolgenden dargelegte Begriff der kulturellen Grenze bezien sationalpolitischen Herr-

(the Policics of the World-Economy: Tormulicrung bei limmanuel Wallerstein, The Pof

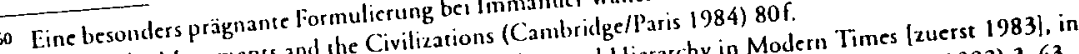

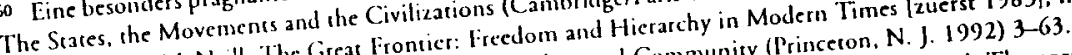
G1 Will ders ins

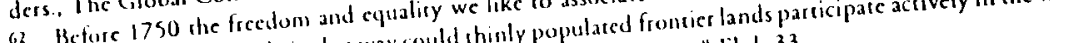

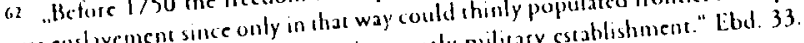

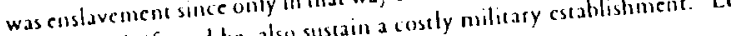
Michel Korinman, Quand Allemagne pensair

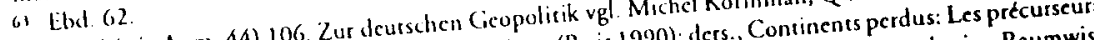
a Ancel (wie Anm. 44) 106. Zur d'une géopolitique (Paris 1990); ders., Col Haushofer und seine Raumwis-

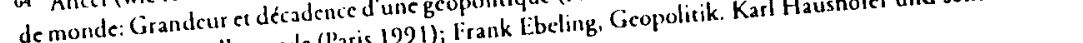

de la géopolitique allemande (Berlin 1994). fassenden Sinne auf das Problem der Konstrukschaftsansprüche, sondern in einem umassenden Simeiten. So gesehen, kann jeder de tion von Differenz zwischen zivilisatorischen Eim Konnotationen kultureller Unterdreiden: Die territoriale Grenze, meist von beiden Seicn hivilisascheidung aufe neuzeitliche Nationalkulturen. Die um vom Limes-Typ ist selber schon geform, Irem gezogene imperiale „Barbarengrenze vom. Die erschließende frontionszentum sichtbarer Ausdruck en tier" treibt im Namen von überlegen Naren, die als Elemente natürlicher Wildnis bedes weißen Mannes schwächere Kuluren, Frontier der europäischen Expansion - also trachtet werden, vor sich her. An der Great Fonsenze - kommt es teils zu so etwas wie der universal verallgemeinerten Erschließungstum sowohl der Europäer wie der "Einheimischen. Ökonomische und politische lnkung einhergehen.

kann mit kulcureller Abgrenzung und Ausschließung einher Scheidelinien: Man überKulurelle Grenzen verlaufen häufig entlang sichibarer Sch Sprachgebiet. Ebenso Kulturelle Grenzen verlaufen hafindet sich in einem anderen Sprachgebiet. Ebenson schreitet eine Staatsgrenze und Grenzen, wie sie sich in Denk- und Vethalcensweiden ist oft sind sie jedoch unsichtbare Grenzen, wiedlungssäumen. Zu unterscheiden ist ausdrücken, nicht aber in Grenzanlagen einer Zivilisation, also ihrer inneren Struktuweiterhin zwischen den Binnengrumen, sozialen Lagen, echnischen Identiär. Krysztof rierung nach räumlichen Verteilungen, sozialen Lag ihren Außengrenzen. Krzysztof rerche gions meint beides, wenn er erklärt: "Die Geschiche Brozeß, der im Zeitalter von Pomian mein "Gs Durch die Expansion Europas, jenen Prozen, der ir der universalen seiner Grenzen. Kreuzzügen und Ostkolonisaikanischer Kulturformen Höhepunkt und Ende ereichVerbreitung europäisch-amerikanischiedes zwischen Europa und Nicht-Europa zu einer te, ist die Bestimmung des Unterschiedes eigener und fremder Identiaten geworden. konstitutiven Frage der Herausbildung enchzudenken, wo sie auf Zivilisaionen Europäer beginnen dort, über sich selbst nachaude ${ }^{2}$ liches verbindet. ${ }^{66}$ Was „EuroEurefen mit denen sie wenig unmittelbar Selbstverstandich. Umgekehrt beschränken trefen, mit denen sie sich erst aus der Kontrasterfahrung. Umg nicht auf die polaren pa " ausmacht, erwer der Bewohner Asiens, Amerikas und Afrikäliger Kapitulation vor sich die Reaktionen der Beffer Abwehr des Europäischen und völliger Kapiculallen NeuMöglichkeiten von schroffer Abwforderung gibt vielmehr oft Anlaß zu kulturellen Nen ihm; die machtgestützte Heraus des Fremden ist Voraussetzung fur die Bewußtwe Vorstlung: Was bestimmungen. Die Erfahrung des Fr ke des Eigenen. Dabei ist „das Fremde keineder Geschiche ent

6s Krzyszof Pounian, Europa und seine Nationen (Benantes. La scule à avoir érige la transgress (1992) 28-47. ropernne ese la seule civilisation des fronnizeres me " L'Europe et ses frontitres, in: Le Debat 68 (1)

Zitar 34 .

66 Vgl. auch Rudolf Wendorf. 413.

politik (Opladen 1984) 413. 
als ,andersartig" und "fremd" wahrgenommen wird, ist nicht anthropologisch festgelegt, sondern kulturspezifisch nach Ort und Zeit variabel: Die Chinesen, um ein deutliches Beispiel zu nennen, sind nicht "gelb", sie werden es allmählich im Auge des frühneuzeitlichen europäischen Betrachters. ${ }^{67}$ In Situationen des Kulturkontakts werden Abgrenzungen vorgenommen, die es zuvor nicht gab; zugleich kann es aber auch zum Abbau von Grenzen und zu Vorgängen gegenseitiger kulcureller Anpassung kom men. „Kulturzusammenstoß" und „Kulturbeziehung", um an Urs Bitterlis Begrifflichkeit zu erinnern, liegen dicht beieinander, sie können koexistieren und vom einen zum anderen abrupt oder allmählich übergehen.

Kulturelle Grenzen stimmen keineswegs immer mit geographischen oder politischen Grenzen, mit "borders“ und „boundaries“, überein. Selbst im Ausnahmefall Japans, wo sich Nationalstaat und Zivilisationsraum zu decken scheinen, hat die Ausgrenzung der auf der Nordinsel Hokkaido lebenden Ainu und ihre Distanzierung als das fremdethnische Andere während der Tokugawa-Zeit eine wichtige Rolle bei der Herausbildung des frühmodernen Staates und seiner Selbstauffassung als Garant ethnischer Homogenität gespielt ${ }^{68}$ Erst recht deckt sich das Europa der Historiker und Zeitbeobachter nicht unbedingt mit dem der Geographen. Schon im Mittelalter rech nete man das Königreich Jerusalem mir seiner mehrheitlich muslimischen Bevölkerung zur christlichen Okumene. ,In der Tat gehen uns Neuyork und Lima näher an als Kiew oder Smolensk", schreibt 1824 der junge Leopold Ranke. ${ }^{69}$ Ranke artikuliert hier mit anti-russischer, auch anti-türkischer Tendenz - ein atlantisches Selbstbewußtsein Amerika nicht als eine ,andere“, eine auch indianisch geprägte Welt, wie sie kurz zuvo Alexander von Humboldt porträtiert hatte, ${ }^{70}$ sondern als eine transozeanische Erweiterung des romanischen und germanischen Europa. Demographisch und kulturell war das nicht unrichtig beobachtet; ökonomisch wird Wallersteins Idee des „modernen Weltsystems" vorweggenommen. Für Reisende des 17. und 18. Jahrhunderts waren die großen Kolonialmetropolen wie Batavia (Jakarta), Mexiko-Stadt oder Kalkutta, von Boston ganz abgesehen, kaum weniger „europäisch“ in ihrem äußeren Erscheinungsbild als osmanische Städte auf europäischem Boden wie Bukarest, Sofia oder Saloniki, wo die Kirchenglocken schwiegen und die Muezzins von den Minaretten zum Gebet riefen. Die klassiziscische Architektur des Hochkolonialismus bekräftigte

67 Vyl. Walter Demel, Wie dic Chinesen gelb wurden. Ein Bcitrag der Frühgeschichte der Rassencheorien, in: Historische Zeitschrift 255 (1992) 625-666

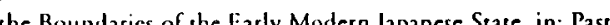
68 $\mathrm{Vgl}$. David L. Howell, Ainu Eethicity and the B

and Present 142 (1ebruar 1994) 69-93, bes. 72-74. 6.) Leopold von Ranke, zit. nach
Ranke (Göringen 1958) 160.

70 Vor allem in seinem lissai sur le royaume de La Nuuvelle Espagne, 3 Bde. (Paris 1811), sowie in Vues de Cordillecres, er monumens des peuples indigenes de l'Amérique, 2 Bde. (l'aris 18:0). Vgl. Charles Minguer, Alexandre de Humboldt: Itisturien el gtographe de l'Amérique espagnole (1799-1804) (Paris 1969) 319ff.; Renate Löschner, Alexander von Humboldes Bedeutung für die Altamerikaniscik, in: Wolfgang-Hagen Hein (Hrsg.), Alexander von Humboldt. Leben und Werk (lrankfure a. M. 1985) 229-262. dann die zumindest symbolische Europäisierung ïherseeischer Brückenköpfe mit sichtbarem Nachdruck.

Umgekehrt beherbergte Europa in seinem geographischen Umriß kulturell Fremdes, zunehmend als nicht-europäisch Aufgefaßtes. Je deutlicher sich eine Identität curopäischer Staaten und Nationen ausbildere und in Spuren auch ein nachmittelalterliches europäisches Gemeinsamkeitsbewußtsein, um so mehr wurden die oft, aber keineswegs immer nicht-christlichen Bewohner der europäischen Peripherie in eine mentale Distanz gerückt, die zuweilen die Bereitschaft zur Gewaltanwendung gegen sie nach sich zog. Dies galt für die Muslime in Spanien bis zur Vertreibung der "Morisken“ 1614 und ebenso - unter ganz anderen machtpolitischen Umständen für die Bekenner des Islam, der namentlich in Bosnien seit dem frühen 16. Jahrhundert eine verbreitete Volksreligion geworden war, auf dem osmanisch beherrschten Balkan. Es galt aber auch für Europas „innere Wilde": tribale oder quasi-tribale Gesellschaften in Randzonen wie Lappland, dem "celtic fringe" (Irland, Schottland, Wales) der britischen Inseln und den östlichen und südlichen Gebieten des Russischen Reiches (Baschkiren, Kalmücken, Krimtataren).

Die kulturellen Außengrenzen Europas lagen also durchaus zum Teil auf dem Kontinent selbst hinter dessen geographischen Umrissen, während sie in Übersee ungefähr den Frontlinien von Besiedlung, militärischer Eroberung und kommerzieller Durchdringung entsprachen. Jenseits solcher Kontaktzonen, die sich vor 1800 in Asien und Afrika mit wenigen Ausnahmen auf Küstenregionen beschränkten und die in Amerika zumindest bis zur Mitre des 18. Jahrhunderts den kontinentalen Kern selten erreichten, ${ }^{71}$ sorgten allein Missionare für eine punktuelle europäische Kulturpräsenz. ${ }^{72}$ So dramatisch aber in den Erfahrungen und Schicksalen einzelner Ordensmänner im kanadischen Urwald, in Japan (bis zum Beginn der großen Christenverfolgungen 1614) oder am Hofe des Kaisers von China die Begegnung zwischen christlich-europäischer Kultur und derjenigen der "Anderen“ ausgetragen wurde, so wenig kann doch von einer "Missionsgrenze" gesprochen werden. Nirgendwo kam es während der frühen Neuzeit außerhalb der (iberischen) Kolonialreiche zu dauerhafter Christianisierung und zur Prägung größerer Kollektive durch weltliche europäische Kultureinflüsse. $^{73}$ Dies änderte sich erst im späten 19. Jahrhundert, als die Mission etwa in China beträchtliche Konversionserfolge erzielte.

1 Vgl. zur Geographie der Erschließung Amerikas durch Europäer jetzt umfassend D. W. Meinig. The Shaping of America: A Geographical Perspective on 500 Years of History. Vol. I: Aclantic America, 1492 1800 (New Haven/London 1986). Eine Übersicht über die Präsenz von Europäern in Asien gibr Holden Furber. Rival Empires of Trade in the Orient 1600-1800 (Minneapolis 1976) 298-329.

72 Vgl. Horst Gründer, Welteroberung und Christentum. Ein Handbuch zur Geschichte der Neuzeit (Gütersloh 1992).

3 Wie stark eine solche Prägung im kolonialen Rahmen nicht nur in Amerika sein konnte, zeigt das fur die Kulturgeschichte der curopäische Expansion überaus instruktive Beispiel der Philippinen. Vgl. Reinhard Wende, Kolonialismus und Kulturwandel. Das Beispiel der Philippinen, Seudienkurs der FernUniversita (Hagen 1992); ders. Kultureller Konflikt, kulturelle Mischung. Die Philippinen unter spanischer und 
Tort ihre Grenze, wo die ihr eigentümlichen Regeln und Fine Kultur erreicht dort ihre Grenze, wo die ihr eigentum nicht länger bestimSymbole die Lebensführung und die Weltbilder der Menschen nich länger ben in die men. Reisende machren diese Erfahrung im Ubergang etwa von der chronialmetropole is misch-osmanische Welt oder dort, wo man eine geschutzte Kolonialmetropole islamisch-osman Ungewißheiten ihres Hinterlandes aussetze. An ihren Grenze verließ und sich den Un (3) 列 nung kann auch in kulturellon Mehrdeutigkeien aufge"ss " wer - charakteristischer mäßig seltenen Phänomen eines religiösen "Synkretsm wie sie in vielen der großen maßig in der Mehrsprachigkeit und den vormodernen Imperien verbreitet waren mehrheitlichen ethnischen Kerngruppe angehorten, verche im Zarenreich, Mongolen cionen in Staat und Gesellschaft aufzusteigen: Deutsche im Zarenton Balkanvölkern unter der mandschurischen Qing-Dynastie in China, Angehö

im Osmanischen Reich, Kaukasier im Iran der Safawiden.

Die kulturelle Grenze trennt vom Fremden. Sie ist meist eine Zone des Obergangs, manchmal eine scharfe Linie. Kuliuren unterscheiden sich zu bestimmen Ze durch ihre durch das Maß an Schrotheit, mit dem sie sich vom Fremden abgeneide terium non Exklusivität. Einige sehen eine radikale Opposition - Christ oder Heide: Maßstäblichkeit datur; andere nehmen Abstufungen von Differenz in Bezi, die fein abgewogene Grade des Eigenen an, so etwa die alte chinesische Hochkultur, die fun des "Be des „Barbarentums unter-Gruppe integrieren und ihnen Möglichkeiten zur kulturelhende mühelos in die Writerien, nach

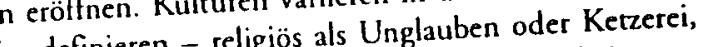
denen sie Fremdes vorzugsweise definieren - religios als säkular-zivilisatorisch als Barbarentum, biologisch als rassische Minderwertigkeit, uswerter sakular-zivilisaiden sich in der Intensität der Widerstände, die sie dem Eintritt Fremder Sie unterscheiden sich in der in den eigenen zivilisatorischen Kreis entgegense Übergänge, Glaubenswechsel. Schließlich lassen Kulturen in unterschiedlichem Maße Ubergan

(in : jiirgen Osterhammel (Hrse), Asien in der Neuzeit. Sieben historische M. 1994) $47-64$

Srationen (Fan (he Late Medieval Castilian-Granadian Frontier, in: Angus Mackay, Religion, Culture and ldeology on 222 Zu den Undeutlichkeiren an dieser Grenze vgl. auch Bartlert/Mackay (wie Anm. 46) 219-243, hier 222. Zu dentier in Al-Andalus: Ideal and Reality, in: Dionisius Eduardo Manzano Moreno, Chrst. The Arab Influence in Medieval Europe (Reading 1994) 83-99. (die vorAgius/Richard Hitchicock (Hele zum Beispiel war (mindestens) dreisprachig: Türkisch, Persisch (die dans 75 Die osmanische Bildungselie bilthafte Sprache der Dichrung) unneran (Hrsg.). Histoire de l'Empire ottoman (Paris IEmpire otcoman, in: Roberten Sprachgebrauchs war das islamische Bosnien. Vgl. Smail Balic

Mikrokosmos solch mulas Brücke zur islamischen Welt (Köln/Weimar/Wien $/$ a) kannte Bosnien. Europas Be Chinesen und das Fremde, in: Helga Breuninger/Renare Bert 1993) 10-18.
76 Vgl. Gudula Linck. Die Che

Umgang mir dem Fremden. Symposium vom 12. bis 14. Juni 1992 schwebende Zwischenzustände, Ambivalenzen oder mehrfache Idenitäen zom Eigenen gängertum und Grenzüberschreitung in der ungekehronktioniert: ${ }^{77}$ Der Abfall vom

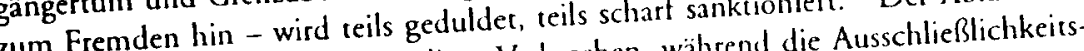
zum Fremden hin - wird Islam galt und gilt als todeswurdiges Veren entwickelt sind. Britische Kolonialherren ansprüche im Buddhismus viel schwächer entwickelt sisten als zum Beispiel portugiehielten eine deutlich größere Distanz zu den Kolonsiertist sische, spanische und selbst holländische: Als Niederlündisch-Ostindien 181 voribert gehend unter britische Verwaltung geriet, zeigten sich die neuen Reglich ihrer Berel über die asiatisierten „Mestizo manners“ der Hollander (und name die sozialen Gräben kauenden Frauen); die Kolonial-Europäer wurden re-zivile zwischen Europäern und Asiaten mit langfristigen Folgen das Ausmaß, in dem sie unterschieden sich die europäischen Kolonisatoren durch das Ausmaßulderen, sowie gemischtrassige Verbindungen und das daraus folgende Mestizentum dulderen, sowie auch darin, ob Geld, Bildung oder Macht den Makel "unteiner Granzosen machen auch darin, Ob Weißen" Indianer zu Spaniern, Afrikaner zu Franzosen machen Farbige sozial zu "Weißen". Indiate sich der Bogen von den in hohem Maße (allerding konnten.79 Pauschal gesagt, spannte sich der Bogen is zu den extrem exklusiven in Asien stärker als in Afrika) inklusiven Portugiesen bis zu dë̈kierung erwa jener Briten, die bis zum Ende der Kolonialzeit von rassistischer Brüskigen relativ günscige anglisierten indischen Oberschicht nicht abließen, der man im üumen bereit war

kulturelle und auch politische Entwicklungschancen einzuräumen bereit war von Kul-

Kulturelle Grenzen sind nich als selbst als Teil einer Kultur deren Artefakte. Deshalb tur natürlich gegeben. Siengen historisch. Dies zeigr die außerordentliche Beweglichkeit verändern sich Abgrenzungen historich von Feindbildern. Es kann Ver-Fremdungen won vremdheit und negativen Wahrben wie auch umgekehrt einen raschen Abure Europas liefert für beides Beispiele. Im nehmungsstereotypen; die jüngere Geschiche Europas im späten 18. Jahrhundert ein nehmungsseräischen Verhältnis zum Rest der Welt ist im späten 18. Jahrindert ein gesamteuropaisch neuartiges, nicht mehr auf religiose Herioriätsbewußtsein zur Geltung gekommen, das sche Uberlegenheit gestütztes Superioritatsbewußsein zur Grühen 19. Jahrhunder überall die kulturellen Grenzen vertiefte. ${ }^{80} \mathrm{Nachdem}$ sich im fruhen 19 . Jist vor allem das von einigen europäischen Intellektuellen - im Cedizhos

- Vol Kravescie der Lebensformen" oder "kulturelle Konversion"? Zur Geschichre des 77 Vgl. Karl-Heinz Kohl, "Travestie der Lebenstornen Verlangen. Zur Geschichte der Ethnologie (Frankfurt

kulturellen Oberläufertums, in: ders., Abwehr und 7-38.
a. M./New York 1987) 7. $78 \mathrm{Vgl}$. Jean Gelman Taylor, The Social Writish Assessments of the Dutch in Asia in the Age of Re (Leiden Wisc. 1983) 100-102, Pence Mas India and Indonesia during the $A$ ncien Reg E. 1400-1715 (London

usw. 1989) $1-13$.
79 Vgl. G. V. Scammell, The First Imperial Age: European Overseas Expansion c. 1400-1715 (London
(1) 1989) $185-190$.

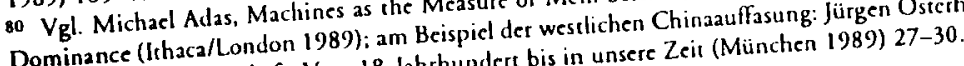

na und die Weltgesellschaft. Vom 18 . 
Goethes Programm der "Weltliteratur" - verfochtene Ideal einer pluralistischen Weltkultur nicht durchsetzen konnte, ${ }^{81}$ kam es zur Antiquarisierung und Musealisierung der orientalischen Kulturen, die sich mit der Abwertung außereuropäischer Gegenwartskultur verband. Im späteren 19. Jahrhundert setzte dann der in manchen kolonialen Milieus virulente Rassismus durch eine verstärkte Biologisierung sozialer Distanzen diese Tendenz zur Abgrenzung zwischen Europäern und Nicht-Europäern verschärfend fort. Dies machte sich nicht nur innerhalb des kolonialen Herrschaftsverhältnisses in den „farbigen" Kolonien selbst bemerkbar, wo sich etwa die Aufstiegschancen von Einheimischen im kolonialen Staatsapparat verminderten, sondern zunehmend auch in den "weißen" Siedlungskolonien, die sich gegen asiatische Überfremdung zur Wehr zu setzen versuchten. Eine hysterische, freilich auch mit politischem Kalkül manipulierte Furcht vor der "gelben Gefahr" erfaßte gegen Ende des 19. Jahrhunderts vor allem pazifische Anrainerländer wie Kanada, Australien sowie Kalifornien und führte dort zu einer Politik der rassischen Exklusion, die wiederum Proteste von Öffentlichkeit und Regierungen in asiatischen Ländern auslöste. ${ }^{82} \mathrm{Als}$ nach dem Ersten Weltkrieg infolge einer langsam einsetzenden Immigration aus den Kolonien der Anteil von Nicht-Europäern an der Bevölkerung der Metropolen allmählich stieg, wurden kulturelle Grenzen und rechtliche Schranken auch in den sich vordem liberal gebenden imperialen Mutterländern - Inder machten immer wieder die Erfahrung, in Großbritannien selbst zuvorkommend behandelt zu werden, nicht aber von den Kolonialbriten in ihrem eigenen Land - verstärkt. ${ }^{83}$ Dies geschah zu einer Zeit der wissenschaftlichen Diskreditierung von Rassedoktrinen; ${ }^{84}$ auf eine rassisch begründete Einwanderungspolitik blieb es ohne Wirkung.

An einer kulturellen Grenze kommt es, wie diese Beispiele zeigen, zu Grenzverhalten: zu Abgrenzungspraktiken oder umgekehrt zur Verringerung einer zunächst großen Distanz. Gesellschaften steht dabei ein Grundrepertoire des Umgangs mit dem Fremden zur Verfügung: ${ }^{85}$

1) seine Inklusion (oder Integration): das friedliche, mit keinem Bekehrungs- oder Anpassungsdruck verbundene Hineinnehmen des Fremden, wie es ist, unter Sicherung seiner Rechtssphäre: gastfreundliche Aufnahme, strukturelle (nicht unbedingt auch gesinnungsmäßige) Toleranz, ethnisch-kultureller Pluralismus;

B1 Vgl. grundlegend: Raymond Schwab, La renaissance orientale (Paris 1950).

82 Vgl. den gesamtpazifischen Überblick bei Avner Offer, The First World War: An Agrarian Interpretarion (Oxford 1989) 164-175, 198-214, sowie fur den gravierendseen Fall Michael Hunt, The Making of a Special Relationship: The Unired States and China to 1914 (New York 1983) 85-114, 227-257.

a) $V_{g}$ l. für Großbritannien Paul B. Rich. Race and Empire in British Politics (2nd ed. Cambridge 1990) 120 ff. Laura Tabili, The Construction of Racial Difference in 20th-Century Britain: The Special Restriction (Coloured Alien Seamen) Order, 1925, in: Journal of British Studies 33 (1994) 54-98.

it Vgl. Elazar Barkan. The Retreat of Scientific Racism: Changing Concepts of Race in Britain and the United States between the World Wars (Cambridge 1992).

ss Ich greife modifizierend ein Schema auf, das Christoph Marx vorgeschlagen hat (in: Breuninger/Breuninger [wie Anm. 76] Anhang).
2) seine Akkomodation: die Herausbildung eines auf gegenseitigem Nutzen beruhenden modus vivendi zwischen selbständig bleibenden Gruppen, die sich lernend aufeinander einstellen, aber ihre jeweilige Identitat im Kern nicht aufgeben;

3) seine Assimilierung. die Angleichung des Fremden an das Eigene bis mitunter hin zur Auflösung einer eigenen Identitär der Fremdgruppe: religiöse Missionierung, weltliche Zivilisierung - nicht immer mit gewaltlosen Mitteln; ${ }^{86}$

4) seine Exklusion: die Abschottung der eigenen Gesellschaft durch Abwehr von Fremden, obrigkeitliche Schließung der Grenzen (so in extremer Form in Japan zwischen 1639 und 1854), scharfe ausländerrechtliche und fremdenpolizeiliche Maßnahmen, Immigrationskontrollen;

5) seine Segregation: die Ausgrenzung des Fremden, seine Isolierung von der einheimischen Umwelt - typischerweise unter Bedingungen rechtlicher und materieller Benachteiligung: also Vertreibung, Zwangsumsiedlung, Ghetroisierung, Apartheid, Einrichtung von Reservaten, usw.; manchmal aber auch in konfliktmildernder Absicht die Einkapselung des Fremden z. B. in Handelsenklaven (wie den chinesischen Treary Ports des 19. Jahrhunderts), die zum beiderseitigen Vorteil den Austausch zwischen inkompatiblen Wirtschaftskulturen ermöglichen;

6) seine Extermination: durch Pogrome und Genozid (z. B. die fast vollständige Vernichtung der Tasmanier oder zahlreicher indigener Völker in Nord- und Südamerika), aber auch - schwächer - Kulturzerstörung durch radikale Zwangsassimilation oder durch Entzug der Chancen für kulturelle Reproduktion der Gruppe („cultural genocide" oder auch "ethnocide"), ${ }^{87}$ ein Schicksal, das bis in die jüngste Vergangenheit etwa den australischen Aborigines widerfahren ist; Amerika und Ozeanien, nicht Asien und Afrika, waren die wichtigsten Schauplätze solcher extremen Entwicklungen.

Jede dieser Formen kann im übrigen eine reflexive Note erhalten, also Selbst-Integration, Selbst-Assimilierung, auch Selbst-Abkapselung als Schurz vor einer feindseligen Umwelt. Im Prozeß der weltweiten Expansion Europas haben Europäer jeden der sechs Grundrypen praktiziert: von der seltenen Haltung einer kulturelle Grenzen auf ein Minimum abflachenden Integrationsbereitschaft bis zum ebenso seltenen anderen

${ }^{26}$ „Assimilierung“ wird hier als Oberbegriff für eine ganze Klasse von asymmerrischen Angleichungsvorä̈nen eingefuhre, ungefähr im Sinne von Raymond H. C. Teske, )r/Bardin H. Nelson, Acculcuration and Assimilation: A Clarification, in: American Ethnologist 1 (1974) 351-367, bes. 365. Oblicher ist die Verwendung von Akkuleuration" als übergeordneter Kategorie. Vgl. die Diskussion bei Friedrich Heck-

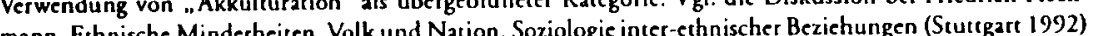
mann, Er hnische Mindereir 167-172, 176-178. Gegen den Begriff der ",Akkuluration spricht nichr allein der babylonische Definitionswirrwarr, der ihn umgibr, sondern auch seine Belastung als Teil der ldeologie eines ,aufgeklarten Kolonialismus in der Zwischenkricgszeit. Vgl. Gerard Leclers, Anthropologie und Kolonialismus (Frankfure
2. M./Berlin/Wien 1976, frz. 1972) 52-61.

67 Leonard Thompson/Howard Lamar. The North American and Southern African Frontiers, in: Lamar/Thompson (wie Anm. 54) 14-40, hier 32. Ein deutliches Beispiel ist die Politik der rotalen Assimilaction, die die amerikanische Bundesregierung zwischen den 1880er und den frühen 1930er Jahren gegenüber den Indianern des amerikanischen Westens betrieb. Vgl. dazu Francis Paul Prucha, The Great Father: The United Intates Government and the Indians, Vol. 2 (Lincoln, Nebr./London 1984) 609 r. 
Extrem der Herstellung von grenzenloser Homogenität durch Auslöschung des Fremden. Zwischenformen und zeitliche Sequenzen verschiedener Formen - etwa von der Akkomodation zum partiellen Genozid in der Erfahrung der nordamerikanischen Indianer - sind für die historische Realität charakteristischer. In manchen Fällen hat die überseeische Welt als Laboratorium für Verhaltensweisen gedient, die später nach Europa re-importiert wurden.

\section{Kontaktsituationen}

Welche der Möglichkeiten des Umgangs mit dem Fremden an der kulturellen Grenze in einem besonderen Fall in Erscheinung tritt, welche also in Verhalten, Handeln oder gar Politik umgesetzt wird, hängt von einer Vielzahl von Faktoren ab, zunächst von den demographischen Proportionen zwischen Selbst - und Fremdgruppe und von den inneren Dispositionen derjenigen Gruppe, die mit dem Fremden umgeht. Wichtig ist daneben die Art der Kontaktsituation, vornehmlich die Machtverteilung, die in ihr manifest wird. Die Spezifik interkultureller Grenzziehung bestimmt sich maßgeblich nach dem, was man das jeweilige Grenzregime nennen könnte. Kulturkontakt ist kaum von den harten Realitäten des Machikonflikts, in den er in aller Regel eingebettet ist, zu isolieren. Im Prozeß der Expansion Europas, vornehmlich während der frühen Neuzeit, findet man vor allem vier typische Situationen: ${ }^{88}$

1) Die Begegnung europäischer Kaufleute, Missionare und Diplomaten mit geschlossenen Reichen ( 2 . B. China, Japan, Korea) führte bis in die Mitte des 19. Jahrhunderts zu verfestigten Fronten, die sich bereits in der physischen Einschränkung der Mobilität der Fremden ausdrückten. Die Gastkulturen wahrten ihre politische Souveränität und ihre symbolische Autonomie, bestätigt in der erzwungenen Befolgung einheimischer Rituale durch die Europäer, die nie anders denn als zeitweilig geduldete Gäste verstanden wurden. Kulturelle Grenzen wurden teils stabilisiert (durch Christenverfolgungen oder durch Verbore, Ausländer die Landessprache zu lehren), teils kontrolliert durchbrochen (die "Hollandstudien", die in Japan am Anfang des 17. Jahrhunderts begannen, im letzten Drituel des 18. Jahrhunderts intensiviert wurden und die erhebliche Kenntnisse über Wissenschaft und Technik des Westens erbrachten). ${ }^{89}$ Die Segregation

\footnotetext{
88 Ausführlicher in anderer Argumentarionsabsicht: Jürgen Osterhammel, Entedeckung und Eroberung, Neugier und Gewalt, in: Christof Dipper/Martun Vogr (Hrsg.). Enrdeckungen und frühe Kolonisation
(Darmstade 1993) 399-429. Weitere Situationen außer den vier wichtigsten müssen unerörtert bleiben: z. B. die Erfahrung von Verschleppung und Kriegsgefangenschaft oder die relativ gewaltarme Begegnung von Forschern und Lifforschren in geographisch-echnologischen Reisen seit dem 18. Jahrhundert.

8.) Vgl. Graw K. Goodman, Japan: The Duech Experience (London/Dover, N. H. 1986) 42ff.; Yoshida Tadashi, Rangaku - Die Hollandischen Wissenschaften, in: Doris Croissant/Lothar Ledderose (Hrsg.), Japan und Europa 1543-1929 (Berlin 1993) 94-106. Die Ambivalenz von Abgrenzung und particller Offnung wird deutlich in den „Neuen Thesen“ des japanischen Konfuzianers Aizawa Seishisai, vgl. Bob Tadahsi Wakabayashi, Antiforeignism and Western Learning in Early Modern Japan: The New Theses of
}

der Fremden konnte gelegentlich durch pragmatische Zugeständnisse seitens der einheimischen Machthaber gemildert werden, so im Handelsstiitzpunkt Macau, der sich freilich nie zu einem Brennpunkt von Kulturtransfer entwickelte. ${ }^{90}$ Kulturelle Grenzen waren als Folge einheimischer Widerständigkeit in diesem Situationstyp klar gezogen. Sie waren aber keine wandernden "frontiers" der Verwestlichung, sondern eher exklusive imperiale „Barbarengrenzen“, die bis zur machıpolitischen „Öffnung" im Zeitalter des Freihandelsimperialismus von den asiatischen Staaten gegenüber dem Westen aufrechterhalten wurden.

2) In semi-permeablen außereuropäischen Ländern, also lockerer strukturierten pluri-echnischen Agglomeraten, wie vor allem den islamischen Imperien der frühen Neuzeit, standen Europäer unter weniger strenger Kuratel. Ähnliches gilt für große Teile Süd- und Südostasiens. Die vorherrschende Form europäischer Anwesenheit war neben einer großen Zahl von Einzelreisenden (wie sie in Ostasien undenkbar war) ${ }^{91}$ - die „Handelsdiaspora“,, 2 die unter Umständen zum Kristallisationskern regionaler wirtschaftlicher Vorherrschaft werden konnte. ${ }^{93} \mathrm{Im}$ islamischen Bereich wurden die alten christlichen Ostkirchen zwar mit dem für Nicht-Muslime vorgesehenen Minderstatus geduldet, es gab aber keinen Spielraum für die katholische oder protestantische Mission. Die religiösen Außengrenzen waren höher, die Barrieren zwischen unterschiedlichen kommerziellen Kulturen niedriger als erwa in China. Wo ethnisch-kulturelle Vielfalt an der Tagesordnung war, wo etwa (wie im osmanischen Reich) Griechen, Armenier oder Albaner bereits eine große Rolle spielten und es eine gewisse Tradition der Integration von erhnisch und religiös Fremden gab (im osmanischen Reich etwa durch die Rekrutierung von Militärkadern aus dem Knabentribut unterworfener christlicher Gtenzbevölkerungen), waren West- und Nordeuropäer weniger unvertraut als in Ostasien. Die geographische Nähe trug zu solcher Vertrautheit bei, der militärische Antagonismus zum Reich des Sultans minderte sie wiederum. Insgesamt geriet für beide Seiten der „choc de mondes“ weniger dramatisch als bei der

1825 (Cambridge, Mass. 1986). Für den weiteren Kontext der Debatten der späten Tokugawa-Zeit vgl. die kommentierten Exzerpte in Ryusaku Tsunoda/W/m. Theodore de Bary/Donald Keene (Hrsg.), Sources of Japanese Tradition (New York 1964) Vol. 2, 84-131, sowie die Analyse bei H. D. Harootunian, Late Tokugawa Culture and Thought, in: Marius B. Jansen (Hrsg.), The Cambridge History of Japan. Vol. S: The Nineteenth Century (Cambridge 1989) 168-258.

$90 \mathrm{~V} !$. Walter Demel, Als Fremde in China. Das Keich der Mitte im Spiegel frühneuzeitlicher europäischer Reiseberichte (München 1992) 52-73.

Reiseberiche (Muther die allein fitr die Zeir vor 1700 a 450 Reiseber ane Yerasimos, Les voyageurs dans l'Empire orroman (XIVe-XVle siècles): Bibliographie, icinéraires er inventaire des licux habites (Ankara 1991) 9.

92 Zum Begriff "trade diaspora” vgl. Philip D. Currin, Cross-Cultural Trade in World History (Cambridge 1984) 1-3. Eine etwas andere Deutung des Phänomens bei Fréderic Mauro, Merchant Communities, 1350-1750, in: James D. Tracy (Hrsg.). The Rise of Merchant Empires: Lang-Distance Trade in the Early Modern World, 1350-1750 (Cambridge 1990) 255-286.

93 Dies zeigr an einem Beispiel Bruce Masters, The Origins of Western Economic Dominance in the Middle East: Mercancilism and the Islamic Economy in Aleppo, 1600-1750 (New York/London 1988). 
Begegnung mit Amerika und dem ferneren Asien im 16. und 17. Jahrhundert. Kulturelle Grenzen waren also nicht so sehr sichtbar demarkierte und statische Umrisse fremder Enklaven (wie in Ostasien) als vielmehr von Fall zu Fall virulent werdende kulturelle Distanzen, die durch den Kosmopolitismus des Fernhandels gemildert wurden. Erst der Philhellenismus des frühen 19. Jahrhunderts konstruierte die islamischtürkische Zivilisation erneut als anti-abendländische Gegenwelt.

3) In kolonialen Herrschaftsverhältnissen ${ }^{94}$ verläuft die offensichtlichste kulturelle Grenze zwischen den kolonisierenden Invasoren und der altansässigen kolonisierten Bevölkerungsmehrheit. Die Kanarischen Inseln im spanischen, Irland im englischen Expansionsbereich bieten dafür die Urbilder der neuzeidichen Kolonialgeschichte. Einen Sonderfall stellen die Immigrantengesellschaften der Karibik dar, deren abhängig arbeitende nicht-weiße Bevölkerung überwiegend aus Afrika zwangsimportiert

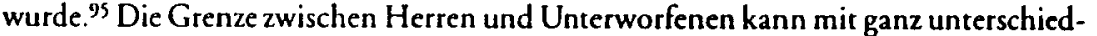
licher Eindeutigkeit gezogen sein. Der Abgrenzungspegel hängt ab von den exklusionistischen oder inklusionistischen Dispositionen der beteiligten Kulturen, ihren relativen demographischen Größenordnungen, von Zweek und Intensität der Herrschaftsausübung. der Art der naturraumlichen l memgebung, der ideologischen Begründung kolonialer Herrschift und den Besichungen der Kolonisierer su ihrem Mutterland. ${ }^{96}$ In der Regel bilden suwohl die Küunisierer als sush die Kolonisierien distinkie, in sich jeweils hiersuchusch geurducte (ieselluhafien, die jedexh an Nahistellen mit-

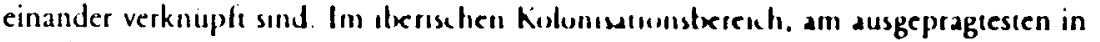
Brasilien, Chile und dem Nordosen Sudamerikss, aber such im Mexiko, entseanden nach langen Integrationsprozessen relativ cinhertlich hierarchisierte "mestizische" Mischgesellschaften, deren Eliten schon in kolonialer Zeit eine (proto-) nationale, die verschiedenen Ausgangselemente überwölbende Kultursynthese propagierten.97 Die $94 \mathrm{Vgl.} \mathrm{dazu} \mathrm{die} \mathrm{definitorischen} \mathrm{Überlegungen} \mathrm{bei} \mathrm{Jürgen} \mathrm{Osterhammel,} \mathrm{Kolonialismus} \mathrm{(München} \mathrm{1995)}$
16. 21.

9s Vgl. die Analysen der karibischen Sklavereigesellschaften bei Franklin W. Knight, The Caribbean: The Genesis of a Fragmented Nationalism (2nd ed. New York 1990) 66-158, sowie David Watts, The West Indies: Patterns of Development, Culture and Environmental Change since 1492 (Cambridge 1987) 326375. Brasilien zeigt Verwandeschaft mir diesem Typ. Ribeiro spriche übergreifend von „neuen Völkern“ ats solchen, "die in den Ietzen Jahrhunderten aus der Vermischung und Akkulturarion von indianischen, afrikanischen und europäischen Elementen als Nebenprodukt der europäischen Expansion entstanden sind" ar Er zählt dazu auch Chilenen und...Groß-Columbianer" (d. h. die Bewohner Kolumbiens und Venezuelas): Darcy Ribeiro, Amerika und die Zivilisation. Die Ursachen der ungleichen Entwicklung der amerikanischen Völker (Frankfurt a. M. 1985, zuerst span. 1969) 108 (Zitar), 350, überhaupt 264-482.

* Ein ähnliches, etwas einfacheres laktorennodell verwender: Robin W. Winks, A System of Commands: The Infrastructure of Race Contact, in: Gordon Marrel (Hrsg.), Studies in British Imperial History: Essays in Honour of A. P' Thormton (London 1980) 8-48, hier $20 \mathrm{ff}$

17 Fur Mexiko zeigr dies Ambony Pagden, Idencity Formation in Spanish America, in: Nicholas Canny/Anthony Pagden (Hrsg.), Colonial Identicy in the Atlantic World, 1500-1800 (Princeton. N. J. 1987) St-93, bes. 6Gif. Line besonders deueliche formulierung der These vom neuartigen Integrationscharakter der mexikanischen Gescllschaft findet sich bei Colin M. MacLachlan/Jaime E. Rodriguez $\mathrm{O}$. The Forging of the Cosmic Race: A Reinterpretation of Colonial Mexico (Berkeley/Los Angeles/London 1980). vor allem auf dem Lande lebende indianische Unterschicht, die zwar in irgendeiner Weise christianisiert war, aber zumeist die spanische Sprache nicht erlernte, blieb jedoch aus dieser gesellschaftlichen Integration großenteils ausgeschlossen. Zwischen der Indiobevölkerung und der spanischen bzw. hispanisierten höheren Gesellschaftshierarchie verlief die tiefste kulturelle Kluft, die Spanisch-Amerika durchzog, auch wenn sie im Lichte neuerer Forschungen nicht als unüberbrückbarer Abgrund gesehen werden sollte: Vielfach bewies die indigene Bevölkerung ein hohes Vermögen zur Akkomodation, ohne eigene Kulturformen assimilatorisch aufzugeben. ${ }^{98}$ Erst die dennoch unübersehbare Dichotomisierung der Kolonialgesellschaft ermöglichte es, daß sich eine Identität des „Indianischen“ herausbildete, wie es sie vor der Kolonialzeit nicht gegeben hatte.

Generell gilt, daß in der Klammer des kolonialen Herrschaftsverbandes separate Gesellschaften fortexistierten, die durch bikulturell kompetente Vermittler, „cultural brokers",99 miteinander in Verbindung traten: Dolmetscher, Missionare, sprachkundige Angehörige der Kolonialverwaltung, kommerzielle Zwischenschichten vom Typus des indischen Banian oder des chinesischen Kompradors, ${ }^{100}$ westlich erzogene "evolués". In sozio-kulcurellen Grenzzonen kam es zu Akkomodations- oder gar Assimilationsvorgängen, deren sinnfallligster Ausdruck die Herausbildung von Kreol- und Pidginsprachen sein dürfte. ${ }^{101}$ Kulturelle Angleichung muß dabei nicht notwendig soziale und ethnische Homogenisierung bedeuten. Sie kann mit zunehmender Beachtung der Hautfarbe, mit der Insistenz auf „racial prestige“ und der Errichtung neuer Schranken einhergehen, wenn sozial aufsteigende Farbige Status, Bildungsprivilegien und Marktpositionen sowohl der kreolischen Oberschicht als auch von „poor whites“ zu bedrohen scheinen. ${ }^{102}$

Kulturelle Grenzen verlicfen auch zwischen Segmenten der kolonialen Untertanenbevölkerung. Nicht wenige Kolonialregime verstärkten nach dem „Divide-et-impera“-

98 "A general principle of Spanish-Nahua (Aztec) interaction is that wherever the two cultures ran parallel, the Nahuas would soon adopt the relevant Spanish form without abandoning the essence of their own form." Lockhart (wie Anm. 16) 243. Die Trennung der beiden Welten wird in der älteren Forschung stärker betont; vgl. resümierend Charles Gibson, Indian Societies under Spanish Rule, in: Leslie Bethell (Hrsg.), The Cambridge History of Latin America, Vol. 2 (Cambridge 1984) 381-419; Mark A. Burkholder/Lyman L. Johnson. Colonial Latin Ameria (New York/Oxford 1990) 190 f.

99 Bernard Bailyn/Philip D. Morgan, Introduction, in: dies. (Hrsg.), Strangers within the Realm: Cultural 92 Bernard Bailyn/Philip D. Morgan, Introduction, in: dies. (Hrsg.). Strangers with

Margins of the First British Empirc (Chapel Hill/London 1991) 1-31, hier 21.
100 Vgl. Peter J. Marshall, Masters and Banians in Eighteenth-Century Calcutra, in: Blair B. Kling/M. N. 100 Vgl. Peter J. Marshall, Masters and Banians in Eighteenth-Century Calcutra, in: Blair B. Kling/M. N.
Pearson (Hrsg.), The Age of Partnership: Europeans in Asia before Dominion (Honuluiu 1979) 191-213; Hao Yen-p'ing, The Comprador in Ninereenth-Century China: Bridge berween East and West (Cambridge, Mass. 1970).

101 Vgl. Suzanne Romaine, Pidgin and Creole Languages (London 1988); Peerer H. Mühlhäuster, Pidgin and Creole Linguistics (Oxford 1986).

102 Für historische Analysen von Situationen dieser Art wären die Überlegungen bei Michacl Banton. Racial and Ethnic Competition (Cambridge 1983) fruchcbar zu machen. 
Prinzip bestehende ethnische und kulturelle Differenzen oder gaben Anlaß zur Entstehung neuer Gegensätze innerhalb von „plural socieries“, in denen ethnische Gruppen auf dem Markt in Wettbewerb miteinander traten. Die Ethnisierung einheimischer Politik ist vielfach erst ein Ergebnis kolonialstaatlicher Eingriffe gewesen: des Ausspielens begünstigter Minderheiten gegen eine Bevölkerungsmehrheit (wie in Burma), ${ }^{103}$ der Trennung zwischen "Stämmen“, die zum Teil erst „erfunden“ oder zumindest zu Verwaltungseinheiten konsolidiert wurden, ${ }^{104}$ und zwischen direkt und indirekt verwalteten Landesteilen, der Vertiefung von Konflikten zwischen religiös definierten "communities" (wie in Indien).

Ebensowenig homogen wie die unterworfene Bevölkerung war die Front der europäischen Invasoren. In Regionen intensiver inter-imperialer Rivalität - der Karibik, Nordamerika, Südostasien, Westafrika - trafen katholische und protestantische Mächte aufeinander und bekämpften sich auch Konfessionsgenossen mit oft großer Brutalität, häufig unter Mobilisierung einheimischer Verbündeter. ${ }^{105}$ Ein Beispiel dafür sind die Allianzen, die sowohl Franzosen als auch Briten seit etwa der Mitte des 17. Jahrhunderts und vor allem während des Siebenjährigen Krieges mit nordamerikanischen Indianerstämmen eingingen. ${ }^{106}$ Die Vorstellung einer natiurlichen zivilisatorischen und/oder rassischen Solidarität der Weißen, wie sie trotz aller machtpolitischen Konflikte bei der Aufteilung Afrikas oder der Niederschlagung des chinesischen Boxeraufstandes (1900) zum Ausdruck kam, ist ein Produkt erst des 19. Jahrhunderts. Kulturelle Grenzen trennten schließlich auch die überseeischen Siedler („Kreolen“ in einem weiten Sinne) von ihren Herkunftsgesellschaften. Häufig bildeten sich besondere koloniale Identitäten unter überseeischen Gruppen in offener oder stillschweigender Abgrenzung von der Kultur des Mutterlandes. ${ }^{107}$ In Fällen einer großenteils absentistischen, die eigenen tropischen Betriebe durch Agenten bewirtschaftenden Herrenschicht, die sich - wie in der britischen Karibik - nicht oder erst spät zu einem stabilen Kreolentum entwickelte, ${ }^{108}$ spielte dies kaum eine Rolle, und in reinen Beherrschungs-

$103 V_{g} l$. Roland Bless, „Divide et impera"? Britische Minderheitenpolitik in Burma 1917-1948 (Stuttgart 1990)

104 Dies ist oft dargestellt worden, besonders deutlich bei John Iliffe, A Modern History of Tanganyika (Cambridge 1979) 318 ff., und neuerdings bei Kurt Beck, Stämme im Scharten des Staats: Zur Entstehung administrativer Häuptlingstümer im nördlichen Sudan, in: Sociologus 39 (1989) 19-35, bes. $25 \mathrm{ff}$.

105 Meinig betrachect in seiner bemerkenswerten geographischen Interpretation des Imperialismus „a complex a is ber plex and unstable fragmentation of every broad tropical region of imperial interest generell als charakteristisch für die frühneuzeitliche Expansion Europas. D. W. Meinig. A Macrogeography of Western Imperialism: Some Morphologies of Moving Frontiers of Political Control, in: Fay Gale/Graham H. Lawton (Hrsg.) Setrlement and Encounter: Geagraphical Studies Presented to Sir Grenfell Price (Melbourne 1969) 213-240. hier 223 .

$106 \mathrm{~V} \mathrm{gl}$. vor aliem francis Jennings, The Anbiguous Iroquois Empire: The Covenant Chain Confederation of Indian Tibes with English Colonies (New York 1984); ders., Empire of Fortune: Crowns, Colonies and Tribes in the Seven Years War in America (New York 1988).

167 Dem ist der wegweisende Aufsateband von Canny und Pagden (wie Anm. 97) gewidmet.

108 Vgl. Edward Brathwaite, The Development of Creole Society in Jamaica, 1770-1820 (Oxford 1971), der Kreolisierung als die Herausbildung einer eigenständigen „grear tradition“ definiert. Vgl, als vorzügliche kolonien ohne ein nennenswertes permanentes Siedlerelement (wie z. B. in BritischIndien) konnte sich allenfalls ein besonderer esprit de corps der Kolonialbürokratie entwickeln. ${ }^{109}$ In Siedlungskolonien hingegen wurde die Abgrenzung von jener Heimatgesellschaft, deren einfache Reproduktion in Übersee zunächst beabsichrigt gewesen war, für die kollektive Bewußtseinsbildung geradezu konstituriv. Immer wieder folgte dabei auf die kulturelle Entfremdung vom Mutterland die politische Sezession. ${ }^{10}$ Dabei verstanden sich die Kolonisten nur ausnahmsweise, wie die neuenglischen Puritaner, als Schöpfer einer utopisch visionierten „new world“; viel häufiger waren sie Träger traditioneller, im Mutterland bereits überlebter politischer Vorstellungen und kultureller Werte: nicht nur in der frühneuzeitlichen atlantischen Welt," auch in den afrikanischen Siedlermilieus des 20. Jahrhunderts - in Kenia, Algerien und zuletzt in Süd-Rhodesien (Zimbabwe). ${ }^{112}$

4) Welche Kontaktvorgänge sich im einzelnen an einer Erschließungsgrenze (,frontier" im engeren Sinne) abspielen, hängt u.a. davon ab, ob es sich um eine von Ackerbau oder Viehzucht bestimmten Siedlungsgrenze (Britisch-Nordamerika, Südafrika, Australien), eine Handelsgrenze (Pelzhandel in Französisch-Nordamerika), ${ }^{113}$ eine Beutegrenze (im Inneren Brasiliens) 114 oder eine andere Variante handelt. Das Voranschieben der Grenze geht oft der Etablierung kolonialer Herrschaftsverhältnisse voraus. In anderen Fällen zieht die Grenzkolonisation die Verdrängung der Einheimischen bzw. die Segregation verbliebener Minderheitsgruppen in Reservaten nach sich. Eine Frontier-Situation unterscheidet sich von einem kolonialen Verhältnis dadurch, daß diejenigen Völker, in deren Lebensraum hinein die Grenze vorangetrieben wird, sich nicht durch direkte Fremdherrschaft in ihrer Existenzweise eingeschränkt sehen. Sie sind, solange sie jenseits der Grenze bleiben, im Prinzip im Besitz ihrer politischen Autonomie, also "frei". Die zersetzenden Wirkungen der vordringenden "weißen" Zivilisation, die die Völker der „Wildnis" vielfach zu unfreiwilligen Agenten weltweiter Handelsinteressen machte, stehen außer Frage. Doch hat es über die Grenze hinweg

Obersicht: Michacl Craton, Reluctant Creoles: The Planters' World in the British West Indies, in: Bailyn/ Morgan (wie Anm. 99) 314-362.

$109 \mathrm{Vgl}$. Philip Mason, The Men Who Ruled India (abridged ed. London 1985) $234 \mathrm{ff}$., sowie jerze der biographische Ansatz bei Clive Dewey, Anglo-Indian Atritudes: The Mind of the Indian Civil Service (London/Rio Grande 1993).

110 Louis Hartz hat in einem einflußreichen Buch von der „Fragmentierung der europäischen Kultur und Ideologie" gesprochen: The Founding of New Socicties: Studies in the History of the United States, Latin America, South Africa, Canada, and Australia (New York 1964) $3 \mathrm{ff}$.

America, So (wie Anm. 97) 267-278, hier 275.

(wie Anm. 97) 267-278, hier 275. 112 Vgl. Dane Kennedy, Islands of White: Serter Society and Culcure in Kenya and Souchern Rhodesia,
$1890-1939$ (Durham, N. C. 1987) 44-47. In diesen Zusammenhang gehört auch dic kulturelle Grenze 1890-1939 (Durham, N. C. 1987) 44-47. In diesen Zusammenhan
zwischen dem burisch und dem britisch geprägten weißen Südafrika."

III Von einer "fur trade frontier" spricht W. J. Eccles, The Canadian Frontier, 1534-1760 (revised ed. Albuquerque 1983) $103 \mathrm{fr}$

114 Vgl. John Hemming, Red Gold: The Conquest of the Brazilian Indians (Cambridge, Mass. 1978) 217ff. 
nicht nur Übernahmen in die jeweiligen kulturellen Codes gegeben, etwa Entlehnungen aus dem Vokabular des Gegenübers, "15 sondern auch pragmatische Anpassungen, welche die konkrete Grenzsituation unmittelbar bestimmten. So haben die neuseeländischen Maori, die chilenischen Araukaner, die Chichimeken im Norden Mexikos und manche Indianervölker Nordamerikas europäische Methoden der Bewaffnung und Kriegfïhrung zeitweise erfolgreich für die eigene Verteidigung einzusetzen vermocht. Umgekehrt wiesen schon früh weitsichtige Kolonialtheoretiker daraufhin, wie wichtig es sein würde, den Einheimischen die Geheimnisse erwa ihrer Landwirtschaft abzuschauen. ${ }^{16}$ Solche Übernahmen erfolgten auf beiden Seiten meist selekriv und in rationaler Auswahl: Indianer in Nordamerika zum Beispiel bemächtigten sich des unmittelbar Nürzlichen, verzichteren aber auf eine umfassende Assimilation und verhielten sich meist ablehnend gegenüber christlichen Missionierungsversuchen, zumal dann, wenn diese - wie die protestanische Mission - einen radikalen Bruch mit dem Herkunftsmilieu verlangten. 117 Erst die "Schließung“ der Grenze und die Immobilisierung der eingeborenen Amerikaner als "captive minority" setzten diese wehrlos den Assimilierungsprogrammen der Weißen aus.

Die neuere ethnohistorische Forschung zur nordamerikanischen Indianergrenze hat das Turnersche Bild eines unvermeidlichen Zusammenscoßes unvereinbarer Lebenswelten in Frage gestellt und umerstrichen, daß nicht a priori eine kulturelle Inkompatibilität von Weißen und Indianem bestand. ${ }^{18}$ Konflikte erwuchsen aus Mißverständnissen und wurden nicht selten auch provoziert. Aus ihrer Eskalation, meist angetrieben von dem Bestreben der Europäer, Situationen unter ihre eigene Kontrolle zu bringen, entwickelten sich dann Feindbilder und verhärtete Stereotype, 119 die wiederum zu Segregation und schließlich beiderseits zum Willen zum Vernichtungskrieg führten, in dem die Weißen obsiegten. Dies geschah schon anfangs des 17. Jahrhunderts in Neuengland und Virgina. ${ }^{120}$ Unter anderen Bedingungen war es aber auch möglich, daß sich in der Art von Urs Bitterlis „Kulturbeziehung“ über längere Zeiträume stabile Machtgleichgewichte einstellten, in deren Schatten es zu Akkomodation, 115 Eine Bilanz solcher kulturellen Wechselwirkungen zwischen Engländern und Indianern zieht James
Axtell, The European and the Indian: Essays in the Eithnohistory of Colonial North America (New York/OxAxrell, The European

116 So Thomas Hariot und die beiden Hakluyts in den 1580er Jahren. Vgl. Karen Ordahl Kupperman, Roanoke: The Abandoned Colony (Savage, Md. 1984) 104

$117 \mathrm{Vgl}$. James H. Merrell, "The Customes of Our Countrcy": Indians and Colonists in Early America, in Bailyn/Murgan (wic Anm. 99) 117-156, hicr 152. 155; James Axtell, The Invasion Within: The Contest of Cultures in Colonial North America (New York/Oxford 1985) 131 ff.

$118 \mathrm{Vgl}$. ciwa Neal Salisbury, Manitou and Providence: Indians, Luropeans, and the Making of New England, 1500-1643 (New York/Oxfurd 1982) 12; Jennings. The Ambiguous Iroquois Empire (wie Anm. 106) 83. 11' Das yroße Gebier der Girenz-Mythologeme und Indianer-Feindbilder erkundet: Richard Slorkin, Regeneration through Violence The Mythology of the American Fironticr, 1600-1860 (Middletown, Cr. 1973) $120 \mathrm{~V}$ Virga Virginia bei James Axtell, The Rise and Fall of the Powhatan Empire, in: ders., After Columbus: Essays in
Assimilation und sogar zur Entstehung einer integriert-inklusiven Grenzzivilisation kam, zur Abflachung kulcurcller Grenzen durch kulturelle Kompromisse. ${ }^{121}$ Derlei konfliktarme Lösungen konnten sich indessen auf längere Sicht nicht behaupten. Der langfristige Trend seit etwa dem späten 18. Jahrhundert begünstigte an fast allen Erschließungsgrenzen des expandierenden Europa die Aufstockung kultureller Barrieren. Ein Ergebnis dieser Prozesse scheint die Entdifferenzierung der Fremdwahrnehmung gewesen zu sein: Die Amerika-Siedler des 17. Jahrhunderts unterschieden noch genau zwischen den einzelnen indianischen Völkern und Gruppen, mit denen sie sich auscinandersetzten. Immer mehr wurde ein solch vielfältiges, lokaler Erfahrung entstammendes Bild durch das abstrakte Klischee des Indianers überdeckt, dem ebenso wie dem Afro-Amerikaner der Anspruch bestritten wurde, Teil der amerikanischen Nation zu sein. ${ }^{122}$ Nicht erst bei Frederick Jackson Turner sah er sich von der Bühne der amerikanischen Geschichte verwiesen und als abschreckendes Emblem von „savagery“ auf eine Statistenrolle reduziert.

Die Kontaktsituaton der Erschließungsgrenze ist, insgesamt gesehen, diejenige mit der weitesten Amplitude von Abgrenzungspraktiken. Im interkulturellen Zwischenreich der Pelzhändler, coureurs de bois, Jesuitenmissionare und indianischen Diplomaten lösten sich die Grenzen zwischen Kulturen auf; von „Kulturbegegnung“ kann hier allenfalls als persönliches Zusammentreffen von Individuen unterschiedlicher Herkunft gesprochen werden. Die Erschließungsgrenze hat aber auch kulturelle Barrieren ermöglicht, höher als in jedem Kolonialsystem. Die weißen "frontier societies" der Neuzeit haben Menschengruppen, deren Arbeitsleistung für die Produktion an der äußersten Peripherie des ökonomischen Weltsystems nicht ausbeutbar war und deren Subsistenzansprüche auf ihre gewohnte natürliche Umwelt dem Landhunger von Siedlern und Bergbaupionieren im Wege standen, in einer Fremdzuschreibung als "Wilde" eingekapselt, ihnen jene minimalen Rechrsgarantien, wie sie fast überall nicht-versklavte koloniale Untertanen genossen, verweigert und ihnen die Chance zur kulturellen Reproduktion genommen. Der „Kulturzusammenstoß" endete in solchen Fällen mit der Ausgrenzung der Unterlegenen aus jeglichem normativen Verständnis von Kultur.

\section{Reflexionsformen kultureller Abgrenzung}

Kulturelle Grenzen an den Rändern der weltweit expandierenden europäischen Zivilisation fanden konkret faßbaren Ausdruck in Sprachverwendung und religiösen Prak-

121 Richard White zeigr dies für das Gebier zwischen Lake Michigan und Lake Superior in seinem Buch The Middle Ground: Indians, Empires, and Republics in the Great Lakes Region, 1650-1815 (Cambridge 1991). 122 Vgl. Robert F. Berkhofer Jr., The North American Frontier as Process and Context, in: Lamar/Thompson (wie Anm. 54) 43-75, hier 45, 49, 52, 61 f. 74; Alden T. Vaughan, From Whitc Man to Redskin: Changing Anglo-American Perceptions of American lindians, in: American Historical Review 87 (1982) 917-953, hier 936. 
tiken, in Bildungseinrichtungen und Formen von alltäglicher Geselligkeit und öffentlicher Kommunikation, ${ }^{123}$ in Festen und Ritualen, 124 in Siedlungsmustern, Stadtanlagen und Architektur, in Zusammentreffen unterschiedlicher Systeme praktischen Wissens (etwa europäischer und einheimischer Medizin), ${ }^{125}$ in unvereinbaren Rechtssystemen samt ihrer realen Auswirkungen, in Weisen der Kriegführung und überhaupt der Konfliktaustragung, usw. Alle diese Aspekte stellen Aufgaben für eine Geschichte interkultureller Beziehungen, die zugleich eine komparative Perspektive nicht außer acht lïßt. Die außerordentliche Vielzahl kultureller Grenzsituationen wäre am einzelnen regionalen Fall zu studieren, der dann wiederum in diachrone Verlaufsmuster und synchrone Vergleichsraster eingefügt werden müßte.

Die Untersuchung interkultureller Perzeptionen fände ebenfalls hier ihren Ort. Durch die leitende Frage nach den Arten und Weisen der Konstruktion kultureller Differenz vor dem Hintergrund tatsächlicher Kontakterfahrungen ließe sich die theorielose Inventarisierung von Textstücken vermeiden, wie sie zahlreiche Studien über die "Spiegelung" fremder Sachverhalte in europäischen Schriften immer noch so unergiebig macht. ${ }^{126}$ Auf diesem Weg wäre der Anschluß sowohl an die neuere Mentalitätsgeschichte als auch an jene Ansätze der, intellectual history" zu finden, die von der Interpretation kanonischer Klassikertexte zur Darstellung quasitranszendentaler „Diskurse" oder rhetorisch bestimmter überindividueller Idiome und Denkstile übergegangen ist.

Es wäre zu beginnen mit der Beobachtung, daß nahezu alle Europäer - selbst die Verfechter eines nach den Maßstäben der jeweiligen Zeit weitgehenden kulturellen Relativismus - seit dem Beginn der überseeischen Expansion um 1500 den Angehörigen anderer Zivilisationen im Bewußtsein eigener Höherwerrigkeit gegenübertraten. ${ }^{127}$ Die gesteigerte Weltoffenheit im Zeitalter der Aufklärung, das zugleich eine Epoche des sich formierenden Rassedenkens und virulenter Rechtfertigungen der

${ }^{123} \mathrm{Zu}$ denken ist hier auch an die verborgenen Gegenöffentlichkeiten der Unterdrückten, wie sie James $\mathrm{C}$. Scott sichebar gemacht har: Domination and the Arts of Resistance: Hidden Transcripts (New Haven/London 1990) 4f. und passim.

$124 \mathrm{~V}_{\mathrm{gl}}$. zur Herstellung von Distanz durch exotisierende Rituale (die selbst wieder aus romantischen Phantasien von curopaischer Höfischkeic genähre wurden) am Beispiel der Staatsaudienzen in Britisch-Indien die musterguiltige Analyse bei Bernard S. Cohn. Representing Auchority in Victorian India, in: Eric Hobs-

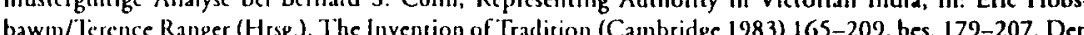
bew umgekehrien Fall, die Anverwandlung cines Kulturamports durch die einheimische Bevölkerung, zeigr am Beispicl der Tiesta: Filipina Wende, Kolonialismus und Kulturwandel (wie Anm. 73) 64-71.

125 Am Beispriel Indicns: David Arnold, Colonizing the Body: Stace Medicine and Epidemic Disease in Ninceenth-Century India (Berkedey/Los Angeles/London 1993) bes. 43ff.

126 Fine Ausuahme an cheoretischer Umsiche ist jecza (iita Dharampal-Frick, Indien im Spiegel deutscher Quellen der Frithen Neuzeit (1500-1750). Studica zu einer interkulcurcllen Konstellation (Tübingen 1994) bor. $1-15$

1:' $V_{g} l$ lur den H humanismus ancli die SchluRfolgerungen bei Wolfgang Reinhard, Missionaries, Humanists and Naures in the Sixcecult. Century Spanish Indies - A Failed Lncounter of Two Worlds? in: Renaissance Studies 6 (1992) 360-376, bes. 376. Die wcrnigen dokumentarisch und literarisch grcißharen Ausnahmen enckräften diese generelle Aussage nichte.
Sklaverei war (bis hin zur Wiedereinführung des Sklavenstatus in allen französischen Kolonien im Juli 1802), hat daran grundsätalich nichts geändert; anschließend wurde im 19. Jahrhundert das größte Ausmaß an „weißer" Distanzierung von den übrigen Kulturen erreicht. Dieses europäische Sonderbewußtsein, welches sein Universalitiatsanspruch über einen beliebigen, selbstverständlichen Ethnozentrismus heraushebt, speist sich ursprünglich aus vorneuzeitlichen Quellen: einer Kombination von hellenischem Barbarendiskurs und christlicher Heilsgewißheit, die später durch neue Bestätigungsstrategien ergänzt oder ersetzt wird: den Stolz auf die eigene Überlegenheit in der wissenschaftlich-rechnischen Beherrschung der Natur, die Überzeugung von der Beglaubigung der okzidentalen Ausnahmestellung durch idealistische oder materialistische Fortschrittsphilosophien, die Selbstzuschreibung eines globalen Zivilisierungsund Modernisierungsauftrags in der Vorstellungswelt des entwickelten Imperialismus. Wie auch immer begründet, beruht das europäische Sonderbewußtsein, das sich in interkulturellen Kontaktsituationen am deutlichsten ausprägt und dort zu realen $\mathrm{Ab}$ grenzungspraktiken beiträgt, auf Ansichten darüber, was im einzelnen die kritische Differenz zwischen dem Eigenen und dem Fremden ausmache. Es hat eine eigene Geschichte, die in der Antike beginnt, sich über die Translatio des Aristotelismus in die Neue Welt neuzeitlich fortsetzt ${ }^{128}$ und mit dem Ende der Kolonialreiche keineswegs abgebrochen ist.

So wäre denn das Problem des europäischen Sonderbewußtseins, wie es sich nirgends so extravagant artikulierte wie in der Kontemplation anderer Zivilisationen, in cine Reihe von Untersuchungsfragen aufzuschlïsseln, die, an Texte wie Reisebeschreibungen, Missionarsrelationen, (proto-) ethnographische Untersuchungen, diplomatische Berichte oder gelehrte und künstlerische Verarbeitungen des primären Beobachtungsmaterials gestellt, zu differenzierten Einsichten in die Reflexionsformen kulcureller Distanzierung und Abgrenzung führen können:

1) Wie fremd ist jeweils das Fremde? Nach welchen Kriterien wird Andersartigkeit bestimmt? Worin sehen Europäer, möglichst mit unmittelbarer Kontakterfahrung, die spezifische Differenz zwischen der beobachteten nichteuropäischen Zivilisation und dem kulturell Eigenen? Worin erblickt man die Besonderheit und immer mehr auch die Überlegenheit Europas? In der Religion, in der Stärke seiner Waffen, im Stand seiner Technik und Wissenschaft, seiner produktiven Kraft, der Zivilisiertheit seiner Lebensformen, in seinen relativ freiheitlichen Zuständen, in der biologischen Beschaffenheit seiner Menschen? Wer wendet diese Kriterien unter welchen Umständen an? Wie verschieben sich über längere Zeiträume die maßgebenden Parameter?

28 Diese Zusammenhänge haben neucrdings viel Interesse gefunden. Vgl. zusammenfassend Wilfried Nippel, Alterrum und Neue Welt, in: ders., Griechen, Barbaren und "Wilde". Alte Geschichte und Sozialanthropologie (Frankfurt a.M. 1990) 30-55, sowic der monumentale Beginn ciner Reihe: Wolfgang Haase/Meyer Reinhold (Hrsg.). The Classical Tradicion in the Americas. Vol. 1, Teil 1: European Images of the Americas and the Classical Tradition (Berlin/New York 1994). 
2) Welches ist die dominante Bezugsgröße individueller Selbstwahrnehmung, etwa des Conquistadors, Reisenden oder Missionars, angesichts einer unmittelbar erfahrenen fremden Umwelt? Fühlt man sich primär - oder in welchem Mischungsverhältnis und mit welchem Rollenrepertoire? - als Christ, „Europäer“, Angehöriger einer bestimmten Konfession, sozialen Schicht, Nation?

3) Mit welchen sprachlich-rhetorischen und bildlichen Mitteln wird die Grenze zwischen Europa und Nicht-Europa bestimmt? Welche „rhétorique de l'altérité wird verwendet? ${ }^{29}$ Welches sind die semantischen Leitposten: „Zivilisation“, „Barbarei“, "Wildheit", „Orient", „Exotik“, usw.? Unter welchen Bedingungen werden solche Vorstellungen, etwa auch als „asymmetrische Gegenbegriffe“, 130 auf eine deskriptive Erfassungweise fremder "Sitten und Gebräuche" projiziert? Werden sie dynamisier (Zivilisierung als transitiver Prozeß, umgekehrt Verwilderung und „Versinken in Barbarei", Orientalisierung und Exotisierung)? Welche Bedeutung wird historischen Bewegungsdifferentialen beigemessen (,geschichtslose Völker“, dynamischer Westen versus stagnierender Osten)?

4) Wie sind Abgrenzungskonstrukte in der Spannung zwischen „Abwehr und Verlangen"131 affektiv beserzt? Paradiesisch-utopische Erwartung und Furcht vor dem Unbekannten; der naturhafte "Wilde" als Projektionsobjekt der eigenen Triebnatur, usw.

5) Welche Theorien europäischer Besonderheit entstehen aus primären Kontakterfahrungen und bei deren sekundärer Verarbeitung durch europäische Gelehrte und „armchair travellers"? Wie werden Differenzen - wertend als Manifestationen europäischer „Überlegenheit“ verstanden - erklärt? Zu denken wäre an frühe ethnologische Systematisierungsversuche angesichts süd-wie nordamerikanischer Indianerkulturen, an Vorstellungen von „asiarischem Charakter" und „orientalischer Gesellschaft", an Rassedoktrinen und Ansätze zu einer politischen Ökonomie der Unterentwicklung.

Dieser letzte Gesichtspunkt läßt sich weiter ausführen, wenn man versucht, die möglichen Reflexionsformen kultureller Abgrenzung in einer unabgeschlossenen Systematik von Diskursen zu ordnen. ${ }^{132}$ Für die Neuzeit wären dabei die folgenden Weisen des Redens über andere Kulturen idealtypisch zu unterscheiden:

Der ethnographische Diskurs, wie er sich bereits bei Herodot findet, ${ }^{133}$ zieht kultu-

129 Der Ausdruck finder sich in einer methodisch vorbildlichen Studie zur „Konstrukrion" von fremdkultu-

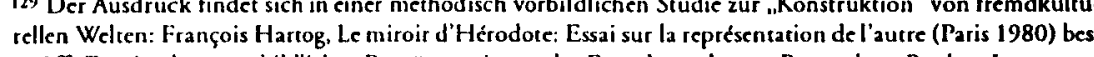
225ff. Zur Analyse von bildlichen Repräsentationen des Fremden vgl. etwa Bernardette Bucher, La sauvage aux seins pendants (Paris 1977), eine Untersuchung der Kupferstiche in Theodor de Brys „Collectiones peregrinationum in Indiam orientalem et Indiam occidentalem" (1590-1634).

160 Reinhart Koselleck, Zur historisch-politischen Semantik asymmetrischer Gegenbegriffe, in: ders., Vergangene Zukunft. Zur Semantik geschichelicher Zeiten (Frankfurt a. M. 1979) 211-259, bes. 218 ff.

$131 \mathrm{~V}_{\mathrm{g}} \mathrm{l}$. den Buchrirel bei Kohl (wie Anm. 77)

132 Erste Überlegungen dazu sind im Gespräch mit Jochen Martin entstanden. Vgl. die Diskussionsvorlage in Breuninger/Breuninger (wic Anm. 76) 188 .

133 Vgl. Wilfried Nippel. Ethnographic und Anthropologie bei Herodor, in: ders. (wie Anm. 128) 11-29, sowic Hartog (wic Anm. 129). relle Grenzen dort, wo Zivilisationen sich durch "customs and manners" in ihrer alltäglichen Lebensführung und in öffentlicher Zurschaustellung unterscheiden. Darunter kann Verschiedenes verstanden werden, fast immer gehören zum Repertoire ethnographischer Aufmerksamkeit aber Fragen nach Nahrung, Kleidung und Wohnung, nach Sexualverhalten und Familienbeziehungen, nach der Stellung von Frauen in der Gesellschaft, der Erziehung der Kinder, nach Formen von Abhängigkeit (besonders Sklaverei), Begräbnisriten, Strafjustiz und der Unterscheidung von sozialen Rängen. ${ }^{134}$ Hervorstechende Eigenarten der jeweils kommentierten Gesellschaft ziehen ein besonderes Augenmerk auf sich: die Jagd- und Kriegsmethoden der nordamerikanischen Indianer, das Kastenwesen in Indien, die herausgehobene Stellung der Gelehrten in China. Der ethnographische Diskurs führt vor dem Ende des 19. Jahrhunderts so gut wie nie zu einer Überzeugung von der völligen Gleichwertigkeit aller Zivilisationen, doch enthält er das Potential zu einer Dämpfung des europäischen Sonderbewußtseins, da sich nach der Art von Montesquieus "Lettres persanes" (1721) die Perspektive umkehren und der ethnographisch distanzierende Blick auf Europa zurücklenken läßt. ${ }^{135}$

Zu den Sitten und Gebräuchen gehören auch Praktiken der Religionsausübung, die sich ohne eine Bewertung der jeweiligen religiösen Gültigkeitsansprüche darstellen lassen. Dies ist bereits im 16. Jahrhundert in der Auseinanderserzung mit den Indianerkulturen Mittelamerikas möglich gewesen ${ }^{136}$ und hat seit der Mitte des 17. Jahrhunderts zu einer umfangreichen quasi-religionserhnographischen Literatur geführt. ${ }^{137}$ Davon zu unterscheiden ist der theologische Diskurs über das Fremde, der in seinen Grundfiguren aus der mittelalterlichen Abwchr der islamischen Herausforderung entstand, aber dann auch offensiv zur Rechtertigung von Kreuzzug und Mission dienen konnte. ${ }^{38}$ Die theologische Erfassung des Fremden muß nicht unbedingt ein schroffer Abgrenzungsdiskurs sein. Viele der rabiatesten anti-islamischen Äußerungen richteten sich nicht gegen den Glaubensgehalt des Islam, sondern gegen den angeblich unmoralischen Lebenswandel des Propheten Muhammad. ${ }^{139}$ In Hochmittelalter war eine Haltung großer theologischer Offenheit gegenüber dem Islam möglich, die auf der

134 Eine für die frühe Neuzeit repräsenrative Systematik läßt sich der enthnographischen Enzyklopädie von Demeunier entrehmen: Jean-Nicholas Demeunier, L'esprit des usages er des coutumes des différens peuples, ou Observations tirées des voyageurs \& des historiens, 3 Bde. (London/l'aris 1776).

$135 \mathrm{Vgl}$ zu dieser Are von Literarur umfassend Winfried Weisshaupt, Europa sicht sich mit fremiem Blick. Werke nach dem Schema der "Lettres persanes" in der europäischen, insbesondere der deutschen Literatur des 18. Jahrhunderts, 3 Bde. (Frankfurt a. M./Bern/Las Vegas 1979).

136 Zuerst bei Bartolomé de las Casas. So Carmen Bernand/Scrge Gruzinski, De l'idolâtrie: Une archéologie des sciences religieuses (Paris 1988) $41 \mathrm{ff}$.

137 Ein Monumenr dieses Interesses ist das aus verschiedenen Quellen kompilierte Werk Bernard Picard, Cerémonies et courumes religieuses de tous les peuples. 8 Bde. (Amsterdam 1723-1743).

Cerémonies et courumes rel igieuses de tous les peuples, 8 Bde. (Amsterdam 1723-174.3). N. J. 1984) bes. 97 f

N. J. 1984) bes. 97ff.
139 Vgl. Norman Danicl. Islam and the West: The Making of an Image (Edinburgh 1960) 79-108 
Hoffnung gründete, die Überlegenheit des Christentums lasse sich den Muslimen in rationaler Argumentation plausibel machen. ${ }^{40}$ Die frühe Neuzeit hat der theologischen Beurteilung des Islam wenig Neues hinzugefügt, sie gegenüber dem Hochmittelalter vielmehr deutlich entdifferenziert und den Islam abermals der Pauschalkategorie des „Heidentums" zugeschlagen. ${ }^{141}$ Überhaupt wurde die Auseinandersetzung mit der islamischen Welt, die sich vornehmlich in Gestalt der türkischen Militärmacht präsentierte, kaum auf theologischem Gebiet geführt. Ein theologischer Umgang mit dem Fremden erlangte dann neue Bedeutung in der Debatte um die verborgenen Glaubenswahrheiten der chinesischen Religion, die seit dem späten 17. Jahrhundert gefuihrt wurde. ${ }^{142}$ Auch hier wieder wurde, wie im Islam-Räsonnement des Hochmittelalters, theologischer Scharfsinn auf den Nachweis kultureller Konvergenzen verwandt. Die gelehrten Missionare des 17. und 18. Jahrhunderts - ob Jesuiten in China, Japan und Amerika, ob dänisch-hallesche Pietisten in Indien ${ }^{143}$ - äußerten sich über die Kulturen, die sie studierten, ohnehin eher in ethnographischer als in theologischer Hinsicht. Die entschiedenste Verdammung des Fremdkulturellen (und damit zugleich die Begründung eigener Besitzansprüche in theologischer Sprache), die es in der Frühphase der europäischen Expansion gab, findet sich bei den Pilgervätern und Puritanern in Nordamerika: Satan benutze die Indianer als seine Werkzeuge. ${ }^{144}$ Erst das „Evangelical Revival", das große Teile der protestantischen Mission in Asien, Afrika und Ozeanien während des 19. Jahrhundert bestimmte und Parallelerscheinungen im katholischen Bereich fand, radikalisierte dann wieder - wenngleich nicht mit der genozidalen Konsequenz puritanischer Extremisten - den theologischen Diskurs zur rigiden Exklusion des verworfenen "Heidentums".

Der juristisch-politische Diskurs drehte sich im Kern um drei Probleme: Sind die Bewohner außereuropäischer Länder vollwertige Rechtssubjekte und halten sie insbesondere gültige Rechtstitel an ihrem Grund und Boden? Wird in diesen Gesellschaften Herrschaft legitim ausgeübt? Unter welchen Umständen sind Eroberungskriege gegen

140 Eiwa bei Roger Bacon und Ramon Lull. Vgl. Richard W. Southern. Das Islambild des Mitrelalters (Sturegart usw. 1981) 45f., 48; Anuenaric Schimmel, Luropa und der islamische Orient, in: Der Islam. Bd. 3: Islamische Kulur - Zeirgenössische Serömungen - Volksfrömmigkeit (= Dic Religionen der MenschBd. 3: Islanische Kultur - Zeigenössische Strömungen - Volksfrömmigkeit (= Die Religionen der Mensch-
heit, Bd. 25/3) (Sturtgart usw. 1990) 336-387, hier 351-353. Vgl. auch den bemerkenswert friedfertigen Kreuzzugskritiker Radulfus Niger und sein Werk De re militari er rriplici via peregrinaroris ierosolimitane (1187/88). Einleitung und Edition von Ludwig Schmugge (Berlin/New York 1977).

141 Man hat desera Umbruch schon auf den fruihen Zeitpunke von 1290 datiert: James Muldoon, Popes, Lawyers, and lufudels: The Church and the Non-Christian World, 1250-1550 (Philadelphia 1979) 52, 69. $142 V_{\mathrm{gl}}$. aus einer selur umfangreichen Liscratur: Claudia von Collani. Die Figuristen in der Chinamission (Frankfurr a. M. 1981): [Rente Eremble, L'Europe chinoise. T. 1: De l'Empirc romain à Leibniz (Paris 1988) $280 \mathrm{HF}$

14! $\mathrm{V}_{\mathrm{gl}}$ zu diesen jerze Heike Liebau, Deuesche Lndienmissionare im 18. Jahrhunderr, Studienkurs der FernUniversität (Hagen 1995); Dharampal-Frick (wie Anm. 126) 95-108, 348-373.

$144 \mathrm{Vgl}$. Roy Harvey P'arce, Rot und Weiß. Die Lirfindung des Indianers durch die Zivilisation (Stuctgart 1991. amerik. 1953) 46-52. diese Völker "gerechte Kriege"? 145 Die meisten Versatzstücke dieses Diskurses, der selbstverständlich wie die bereits genannten ebenfalls antike Grundlagen hat, wurden bereits im 16. und frühen 17. Jahrhundert ausgebreiret: in den Amerikadebatten der spanischen Spätscholastik, ${ }^{146}$ in den Anspriichen der englischen Kolonisten auf land eigentum und Souveränitätsrechte in Nordamerika, ${ }^{147}$ in den Begründungen des englischen Dominium über das gälische Irland. Das anglo-irische Beispiel ist deshalb besonders interessant, weil hier an der innereuropäischen Peripherie die Demarkation einer kulturellen Kluft, teilweise ethnographisch begründet, zwischen dem gälischen Recht der Iren, also dem Brehon Law, und dem englischen Common Law durch englische Kronjuristen unmittelbar zur Rechrfertigung umfassender kolonialer Landenteignungen benutzt wurde. ${ }^{148}$ Juristisch-politische Diskurse sind offenbar meist dichotomisierender Natur gewesen. Die Gegenüberstellung von außereuropäischen kollektiven und europäischen individuellen Eigentumskonzepten hat ebenso bis ins frühe 20. Jahrhundert hinein eine große - auch kolonialpolitische - Rolle gespielt wie die Opposition zwischen den legitimen Herrschaftsformen der europäischen Tradition und der angeblich illegitimen ,Despotie" in Asien und Afrika.

Ein historischer Diskurs ${ }^{149}$ wird überall dort geführt, wo fremdkulturelle Gesellschafren in Beziehung zu Entwicklungsmodellen gesetzt werden. Er wird in ausgeprägter Form erst möglich, nachdem es zu einer Säkularisierung der universalhistorischen Auffassung gekommen ist. Die fundamentale Grenzziehung ist hier diejenige, welche die Sphäre des „Historischen" umschließt. Daraus ergibe sich dann die Ausgrenzung einiger oder der meisten nicht-okzidentalen Gesellschaften als „Völker ohne Geschichte". Diese Exklusion gewinnt erst im 19. Jahrhundert größeres Gewicht. Bis dahin ist das frühneuzeitliche europäische Geschichtsverständnis, wie es bei Autoren der französischen und schortischen Aufklärung in Stadienmodellen der universalen Gesellschaftsentwicklung seinen Höhepunkt erreicht, eher inklusiv und berücksichtigt prinzipiell alle in der Geschichte bekannten Gesellschaftsformen. Eine Zwischenstufe der

145 Grundlegend für viele Facetten dieses Diskurses ist Jörg Fisch, Die curopäische Expansion und das Völkerrechr. Die Auseinandersetzungen um den Status der übersecischen Gebiecte von 15. Jahrhundert bis zur Gegenwarr (Sturtgart 1984); vgl. auch ders., Völkerrechutiche Verträge zwischen Spaniern und Indianern. in: Jahrbuch für Geschiche, Staar, Wirrschaft und Gesellschaft Lateinamerikas 16 (1979) 205-244.

146 Vgl im Überblick Reinhard (wie Anm. 13) Bd. 2, 64-67; Oto Kimminich. Die Entstehung des neu$146 \mathrm{Vgl}$ im Uberblick Reinhard (wie Anm. 13) Bd. 2, 64-67; Otto Kimminich, Die Entstehung des neuzeitlichen Völkerrechts, in: Iring Ferscher/Herfried Münk
Ideen. Bd. 3 (München/Zürich 1985) 73-100, hier 79-90.

Ideen. Bd. 3 (München/Zürich 1985) 73-100, hier 79-90.
147 Vgl. Hermann Wellenreuther, Land. Herrschaft und Alleag. Die Indianer und die englischen Kolonien $177 \mathrm{Vgl}$. Hermann Wellenreuther, Land. Herrschaft und Alleag. Die Indianer und die englischen Kolonien
vor der amerikanischen Revolution, in: Hans Thomas (Hrsg.). Amerika - eine Hoffnung, zwei Visionen (Herford 1991) 213-243, bes. 214-223.

148 Vgl. Hans S. Pawlisch, Sir John Davies and the Conquest of Ireland: A Study in Legal Imperialism (Cambridge 1985): Ronald Asch. Kulcurkonfliks, koloniale Herrschafe und Konfession in liland (ca. 15341660). Seudienkurs der FernUniversitär (Hagen 1995).

19) Vgl. Jürgen Osterhammel, Neue Wele'tn in der curopäischen Geschichessschreibung (ca. 1500-1800). in: Wolf Kürler/jurn Rüsen/Ernst Schulin (Hrsp). Geschichrstiskurs II (Frankfure a. M. 1994) 202215 . 
Abgrenzung ist die Unterscheidung zwischen "jungen“ und „alten", zwischen stagnicrenden oder gar niedergehenden und dynamischen Völkern, zwischen sterilen und kreativen Kulturen. Sie läßt aber die Möglichkeit von Wiederbelebung und Zivilisierung offen, die den als vollkommen "geschichtslos" disqualifizierten menschlichen Gemeinschaften abgesprochen wird.

Ein biologisch-rassischer Diskurs schließlich, ${ }^{150}$ dessen Ursprung in der Antike strittig ist, ${ }^{151}$ geht in seinen Sachaussagen über den Bereich kultureller Abgrenzungen hinaus, postuliert er doch die Existenz von Unterschieden, die vorkulturell gegeben und kulturellen Einwirkungen entzogen sind. Ein Denken in biologischen Rassekategorien gewinnt dort an Virulenz, wo nicht länger nur innerhalb von Gesellschaften einzelne Gruppen nach ihrer Hautfarbe unterschieden werden, wie dies mit einer zunehmend verfeinerten Terminologie der Blutreinheit und Blutmischung erwa im spätkolonialen Mexiko geschah, sondern ganze Zivilisationen auf ihren unveränderlichen biologischen „Rassecharakter" als ihre alles determinierende Grundeigenschaft reduziert werden. Sobald dies geschieht, ist der Schritt vom vormodernen Rassedenken zum modernen exklusionistischen Rassismus getan.

Diese fünf Diskurse - und womöglich noch andere, die hinzuzufügen wären bilden das Grundrepertoire von Reflexionsformen kultureller Abgrenzung. Mehrere von ihnen können sich in einem einzigen Text zusammenfinden; manche der großen Reisebeschreibungen etwa kombinieren Elemente aus verschiedenen Redeweisen über das Fremde. Es lassen sich separate Geschichten der einzelnen Diskurse schreiben, doch manchmal um den Preis der willkürlichen Auflösung komplexer Gemengelagen. Es sollte sogar möglich sein, die epochale Dominanz einzelner Diskurse zu ermitteln: vielleicht des juristisch-politischen im 16. und 17., des ethnographischen und historischen im 18. und frühen 19., des biologisch-rassischen im späteren 19. Jahrhundert. Auch kann gefragt werden, welche Erfahrungen Anlaß zum Rückgriff auf welchen Reflexionstyp gaben. So fält auf, daß ein ethnographisches Interesse früh in Amerika geweckt wurde, dann in der Völkervielfalt Süd- und Südostasiens Ansatzpunkte fand und schließlich die Südsee und Afrika zu Schwerpunkten erkor, daß ein biologischrassisches Interesse sich zuerst an die schwarzen Afrikaner knüpfte (auf ihrem Heimatkontinent ebenso wie in der Neuen Welt), daß der Islam und später der Buddhismus zu besonders wichtigen Herausforderungen für die Theologie wurden, während sich das osmanische Reich samt seinen europäischen und nordafrikanischen Säumen, den klassischen Ruinenländern Griechenland und Ägypten, im 18. Jahrhundert als klassi-

151" Er nuß miche in jedem liall auch rassistisch sein, also deskripriv erfaßte Unrerschiede der menschlichen Physis wertend und hierarchisicrond interpecticeren. Die Abolitionsbewegung zum Beispiel argumentierte ceilwrise auf Jerselben biologisch-anthropologischen Lbene wie die Befiurworter der Sklaverei. Vgl. Philip reilwcise auf Jerselben boulogisch-anthropologisch action, 1780-1850 (Madison, Wisc. 1964) 228 ff.; David D. Cutsin, The lmage of Africa: British ideas and Action, I780-1770-1823 (Ithaca/London 1975) 303. ist V View of Blacks (Cambridge, Mass. 1983). sches Beispiel für "Rückständigkeit" und "Niedergang" in ein Lieblingsobjekt für historisch-geschichtsphilosophisches Räsonnement verwandelte.

Es ist in diesem Aufsatz vorgeschlagen worden, die Expansion Europas, deren Ursachen und Ursprünge ohne eine eingehende sozial- und wirtschaftgeschich diche Untersuchung unverständlich bleiben müssen, in ihren Wirkungen auch - und mit Nachdruck - als eine kulturelle Erscheinung zu begreifen. Darunter ist nicht unilateral und unilinear die weltweite Verbreitung europäischer Kulturformen von Religion und Sprachen bis hin zu Rechts- und Erziehungssystemen zu verstehen, sondern ein Kontakt zwischen Menschen mit unterschiedlichem Zivilisationshintergrund, der nur ausnahmsweise in den extremen Formen dauerhaft friedlichen Zusammenlebens oder totaler Überwältigung der einen durch die andere Seite vonstatten ging. Jeder einzelne ganz konkrete Fall von Kulturkontakt kann in dem Maße, wie die Quellen dies zulassen, ,dicht" beschrieben werden. Dies wird weiterhin die vordringlichste historiographische Aufgabe sein. Sogar dazu aber und erst recht für jeden Versuch, von einer höheren Warte aus, die nicht unbedingt die grandiose des "Weltsystems" zu sein braucht, die Vielfalt der in der Geschichte realisierten Möglichkeiten zu ordnen, ist eine Begrifflichkeit höheren Allgemeinheitsgrades als desjenigen der Beschreibungssprache erforderlich. Urs Bitterlis typologische Unterscheidung von drei oder vier Hauptformen der „Kulturbegegnung" bieter dafür eine solide, aber verfeinerbare Grundlage. Das Konzept der kulturellen Grenze, bei dem sich Vorstellungen aus Ethnologie und Soziologie mit Ideen aus Diskursanalyse, historischer Geographie und neuerer Expansionsforschung verbinden, prizisiert die Idee des Kulturkontakts, indem es voraussetzt, daß Zivilisationen sich (zunächst) nur mit ihren jeweiligen Peripherien berïhren, und dynamisierr sie, indem es feine Gradierungen des Aufbaus und Abbaus von Grenzen zuläßt. Aus Bitterlis eindimensionaler Grundtypologie - Kulturberührung, Kulturzusammenstoß, Kulturbezieliung, Kulturverflechtung - wird ein dreidimensionales Skalensystem. Die drei Dimensionen sind 1) die der Abgrenzungspraktiken in der Bandbreite zwischen Inklusion und Extermination, 2) die der Kontaktsituationen im Spektrum von igelhafter Abwehr zu offenen Frontier-Verhältnissen und 3) die der Reflexionsformen kultureller Abgrenzung, wie sie am deutlichsten in Gestalt von literarisch dokumentierten Diskursen erkennbar sind.

Der Zweck dieses Versuches ist erfüllt, wenn sich die vorgestellte Terminologie als mindestens partiell - nicht notwendigerweise in toto - brauchbar erweisen sollte. Zugleich sind damit aber auch Voraussetzungen einer Theorie der globalen Kulcurbeziehungen in der Neuzeit vorgeschlagen worden, obschon noch nicht diese Theorie selbst. Falls eine solche Theorie erstrebenswert sein sollte, bliebe nach ihren Ansprïchen und ihrer Form zu fragen. Sie könnte womöglich eine evolutionistische Theorie sein, gewissermaßen ein kulturwissenschaftliches Pendant zu Immanuel Wallersteins Lehre von der ökonomischen Entfaltung eines modernen Weltsystems. Sie könnte aber auch - und dies wäre vorzuziehen - bescheidener bemessen werden. Dann wäre es das Ziel theorecischer Überlegungen, aus der kombinierenden Zuordnung von mindestens zwei der drei Dimensionen zueinander universalgeschichutlich bedeutsame kulturelle 
Konfigurationen herauszumodellieren und ihre Metamorphosen in der Verschiebung

der Parameter zu beobachten. 